\title{
Temporal course of perceived auditory duration
}

\author{
DOMINIC W. MASSARO and WENDY L. IDSON \\ University of Wisconsin, Madison, Wisconsin 53706
}

\begin{abstract}
A series of experiments explored the temporal course of the perceived duration of an auditory stimulus. A backward recognition masking paradigm was utilized, in which the subject was to determine which of two target durations was presented on a given trial. The target tone was followed, after a variable silent intertone interval, by a masking tone which could assume one of three possible durations. Identification of the long target was found to improve consistently with increases in the intertone interval. In contrast, identification of the short target was as accurate at the short as at the long intertone intervals. Blocking the intertone interval across experimental sessions eliminated these differences between the two target tones. Performance on both the long and the short target tones improved monotonically with increases in the intertone interval. When masking tone duration was randomized within an experimental session, identification of the long (short) target was most accurate with the longer (shorter) masking tone. Blocking the duration of the masking tone across experimental sessions eliminated the effect of the duration of the mask, but did not alter any of the other results. A model, proposed to account for the results, assumes that the percept of target duration grows over time, and can be terminated by the onset of the masking tone. The masking tone also acts to lengthen the perceived duration of the target tone, and this lengthening is a direct function of the duration of the masking tone.
\end{abstract}

In both psychophysics and perception, a great deal of research has been devoted to analyzing such psychological attributes of sound as pitch, loudness, and localization. Yet, surprisingly little effort has been directed toward what would appear to be an equally important attribute, perceived stimulus duration. This empirical gap does not appear to be unique to audition, an equally small amount of research having been conducted on duration in the visual modality. Though substantive theoretical work has been done (Allan \& Kristofferson, 1974a; Creelman, 1962), the manner in which duration is perceived and remembered remains to be resolved. The intent of the current paper is to provide a theoretical model for perceived duration that is compatible with a more extensive theory of auditory recognition (Massaro, 1975b). In order to accomplish this, it will first be necessary to review briefly the critical issues concerning perceived duration (cf. Allan \& Kristofferson, 1974b, for a more extensive discussion). As duration studies employing both visual and auditory stimuli have yielded comparable results (Allan \& Kristofferson, 1974b),

This research was supported by Public Health Service Grant MH-19399, and was conducted while W. L. Idson was supported by a University of Wisconsin Graduate Traineeship. The work represents a collaborative effort; order of authorship is arbitrary. The authors would like to thank the members of the Wisconsin Human Information Processing Program (WHIPP), especially David Klitzke, for helpful comments. The helpful reviews of A. B. Kristofferson, E. A. C. Thomas, and an anonymous referee are also acknowledged. Requests for reprints should be sent to Dominic W. Massaro, Department of Psychology, University of Wisconsin, Madison, Wisconsin 53706. work from the two modalities will be considered together, though the emphasis will be placed on audition.

An initial question which must be raised is whether perception of duration can be conceptualized in the same way as perception of attributes such as frequiency and intensity. The psychological impression of pitch is primarily based on frequency, whereas amplitude is the primary determinant of perceived loudness. In contrast, perceived duration may not be based directly on temporal extent. As stimulus duration covaries with intensity, duration could be inferred from an analysis of stimulus energy. However, empirical investigation has not shown intensity to play a critical role in perceived duration. Research employing a two-interval forced-choice task for duration discrimination has shown that for blank intervals surrounded by clicks, noise-filled intervals, or tone-filled intervals, changes in intensity had no effect upon the discrimination of duration, within the range of .63 to $640 \mathrm{msec}$ (Abel, 1972a, b; Creelman, 1962). The only constraint on this finding was that stimulus intensity had to be sufficient to allow good detectability. When the signal-to-noise ratio of intervals was high, discriminability of duration remained constant over intensity changes. With a low signal-to-noise ratio, however, performance was found to improve with increases in intensity (Creelman, 1962). It seems reasonable to conclude, then, that duration is perceived directly from temporal extent (Allan \& Kristofferson, 1974b).

The next question of interest concerns the manner in which perception occurs. Two principal models have been proposed (Allan, Kristofferson, \& Wiens, 
1971; Creelman, 1962). The earliest model was suggested by Creelman (1962) to account for data from a two-interval forced-choice task. Creelman's model maintains that during presentation of a stimulus of a given duration, the subject accumulates pulses. These pulses are assumed to derive from a set of independent elements, each having a fixed probability of firing. The distribution of pulses for a given duration, $\mathrm{d}$, is approximated by a normal distribution with a mean and variance equal to $\lambda d$, where $\lambda$ is a constant representing the rate of firing. In the forced-choice task, the number of pulses for the second stimulus is subtracted from that for the first stimulus in order to determine the interval containing the longer stimulus. When the first interval contains the longer stimulus, the result will be a normal distribution with a mean of $\lambda d$. The resulting normal distribution will have a mean of $-\lambda d$ when the first interval contains the shorter stimulus. Creelman assumed that the subject would respond that the first interval contained the long stimulus when the output of the subtraction exceeded some criterion value.

Creelman's model (1962) had the virtue of being compatible with signal detection models of processing other dimensions of the stimulus (Green \& Swets, 1966). Allan and Kristofferson (1974a) have argued, however, that this is precisely why it was unable to account for much of the subsequent data. In fact, they suggest that no traditional psychophysical or signal detection model will be able to account for duration discrimination. They base their argument largely upon three discrepancies between the results of studies of duration and similar studies of other stimulus attributes (Allan \& Kristofferson, 1974a, b). The first of these discrepancies concerns the effect of interstimulus interval (ISI) in the two-interval forced-choice task. Research on pitch, loudness, visual location, and visual movement has found that discriminability decreases over increases in ISI. In contrast, when the task is to discriminate the duration of short stimuli, performance improves as the ISI is increased to about $1 / 4$ to $1 / 2 \mathrm{sec}$ (Carbotte, 1973; Small \& Campbell, 1962), and remains fairly constant out to a 2,000-msec separation (Allan, Kristofferson, \& Rice, 1974). The second discrepancy concerns comparative performance on two-interval forced-choice and single-interval absolute-judgment tasks. Research with a variety of stimulus attributes consistently shows superior performance in the forced-choice task, a result predicted by most sensorydecision models (Green \& Swets, 1966). However, studies which explicitly compared duration discrimination in these two paradigms found performance to be quite similar (Allan \& Kristofferson, 1974c; Allan et al., 1974; Carbotte, 1973). The third discrepancy concerns the effect of absolute duration on discriminability. For example, as $\lambda$ is a constant in Creelman's model, both the mean and the variance of the subjective duration of the stimulus should increase directly with actual stimulus duration. This has the effect of predicting that discriminability in the forced-choice task will decrease with increases in the duration of the stimuli, provided that the difference between the stimuli is held constant; a prediction which has not received empirical support (Allan \& Kristofferson, 1974c).

On the basis of such results, Allan and Kristofferson $(1974 a, b)$ argues that a model for discrimination of duration must differ from current psychophysical or signal detection models. Allan and Kristofferson (1974a) offer a two-stage model specifically for duration which can account for most of the available data. In the first stage, it is assumed that objective duration is mapped into subjective duration by means of a quantal mechanism. The assumption is made that the variability of the internal duration is independent of the stimulus duration. The variability results from variation in the onset and offset times of the internal stimulus. For any stimulus, the internal onset-offset times are independently and uniformly distributed over the range of $\mathrm{q}$ msec. This yields a triangular distribution of subjective durations, $f_{i}(I)$ spanning $2 q$ msec, with a mean equal to the objective duration and a variance, $q^{2} / 6$, which is independent of the duration. In the second stage, it is assumed that the subject compares each of the two stimuli to a criterion and makes independent decisions about their durations (long or short). The outcomes of these two categorizations are compared in determining the response. Given that the subject categorizes the stimuli as long or short, the model predicts equivalent performance in the forced-choice and absolute-judgment tasks. Moreover, given that the subjects have sufficient time to make a decision concerning the first stimulus, no effect of the interstimulus interval would be expected. The model also predicts that performance should be a function only of the difference in duration between the two stimuli in a forced-choice task, not of their absolute durations. Thus, the onset-offset model can account for the three principal discrepancies between studies on duration and those on other dimensions.

Though the onset-offset model is capable of accounting for most of the relevant data, it assumes that there is something special about perceived duration which nessitates an independent model. However, the need for such an assumption depends upon the weight given similarities and differences across experimental situations. The effect of decreased discriminability with increases in ISI depends upon a number of experimental conditions, regardless of the stimulus attribute being tested. A critical task consideration is whether the observer compares the two stimuli in the delayed comparison task on the basis of perceptual or categorical codes (Massaro, 1975b, pp. 479-481). If only a few standards are used, the observer is capable of learning to categorize these 
stimuli in an absolute sense. In this case, the observer does not compare his perceptual memory of the perceived duration of the standard tone to the perceived duration of the comparison tone, but compares some abstract categorization, such as "long" or "short" of the standard tone with the categorization of the comparison tone. Given that the subject has no problem remembering the categorization of the standard tone, no forgetting should occur with increases in ISI. The fixed-standard procedure of Allan et al. (1974) would encourage categorization of the stimuli, which might eliminate any forgetting with increases in ISI. In addition, one cannot always expect decreased discriminability with increases in ISI even if the observer is forced to base his judgment on perceptual rather than abstract codes (Deutsch, 1970; Massaro, 1975b). For example, Massaro (1970b) found a significant decrease in memory for pitch with noise- or tonefilled ISIs but not with blank (silent) intervals. Carbotte's (1973) failure to find significant forgetting with increased ISI in the duration task may be due to the employment of blank intervals between the standard and comparison stimuli. This analysis shows that previous results could be primarily a function of particular task conditions rather than implying anything unique about processing the attribute of stimulus duration.

Moreover, there is a noticeable parallel between one aspect of the effects of ISI on duration discrimination and discrimination of other stimulus attributes. This is the finding that in a forced-choice task, discrimination is disrupted when the shortduration stimuli are separated by ISIs less than 250 msec (Carbotte, 1973; Small \& Campbell, 1962). Massaro and Idson (Note 1) modified a backward recognition masking task (Massaro, 1970a) in order to explore relative pitch judgments. Two $20-\mathrm{msec}$ tones were presented on each trial, separated by a variable silent ISI within the range of 5 to $505 \mathrm{msec}$. The frequency of the first tone was chosen randomly on each trial, and the frequency of the second tone was slightly higher or lower than the first. The subjects' task was to determine whether the second tone was higher or lower in pitch than the first tone. Performance improved consistently with increases in the ISI asymptoting at roughly a 200 -msec separation between tones. This result is highly consonant with the decrements in duration discrimination performance in a forced-choice task, with short stimuli and short ISIs (Carbotte, 1973; Small \& Campbell, 1962).

In contrast to studies of other stimulus attributes, Allan et al. (1974) failed to find a significant difference between performance in a forced-choice and absolute-judgment task. One might argue that this involved a weak between-subject comparison, but Carbotte (1973) has found the same results in a within-subject design. If subjects categorize the standard and comparison stimuli in the forced-choice task, performance will not improve relative to the absolute-judgment task. If the forced-choice task is designed to encourage the utilization of perceptual codes, however, we predict that the relative comparison available in the forced-choice task will produce superior performance to that found in the absolutejudgment task. If this prediction is supported, duration discrimination will share another property common to discrimination of other auditory attributes.

The final difference concerns the argument that absolute stimulus duration does not always affect duration discriminability, a clear violation of Weber's law. As Allan and Kristofferson (1974b) point out, however, this finding is not universal. Allan et al. (1974) found a large decrease in discriminability when the absolute duration was increased from 50 to $100 \mathrm{msec}$. Massaro and Kahn (1973) found that judgments of durations of brief lights and tones were critically dependent on the absolute stimulus duration. Discriminating lights and tones of 40 and $100 \mathrm{msec}$ was much easier than discriminating these stimuli at durations of 100 and $160 \mathrm{msec}$, respectively. It could be that the just noticeable difference for stimulus duration, like other stimulus attributes, is relatively constant within a small range of stimulus durations so that Weber's law will not always hold.

Our analysis of the evidence reviewed by Allan and Kristofferson (1974b) shows that duration may not be a unique attribute of sound. There is, however, a body of research conducted from a somewhat different perspective, which suggests that the argument for the uniqueness of duration may be valid. The experiments reviewed above were primarily concerned with a discrimination, or information, measure of perception. The measure of performance in these studies has simply been the extent to which an observer can reliably discriminate Event $\mathbf{A}$ from Event B. Such a measure neither provides, nor is it intended to provide, a direct index of the perceptual experience of duration. In contrast, two sets of studies (Efron, 1970a, b, c; Gol'dburt, 1961) have concerned themselves explicitly with perceived duration. Research which has investigated perceived duration in this manner has found it to vary as a function of two principal factors, the duration of the tone itself and the duration of the following silent interval. Efron $(1970 a, b, c)$ presented a short sound and a light and the observers were first asked to judge when the onset of the light occurred simultaneously with the onset of the sound. They were then asked to judge when the onset of the light occurred at the offset of the sound. Efron was able to determine the subjective duration of the sound stimulus on the basis of these two judgments. The point at which 
the onset of the light and the onset (offset) of the sound appeared to be simultaneous can be taken as the subjective onset (offset) point of the sound, allowing computation of perceived duration. The principal finding of these studies was that accurate perception of duration occurred only for stimuli longer than $130 \mathrm{msec}$. For sounds less than this length, the actual duration was overestimated to produce a perceived duration of roughly $130 \mathrm{msec}$. Efron interpreted this finding to mean that there is a minimal time, on the order of $130 \mathrm{msec}$, required for a perception to occur.

In addition to varying with actual duration, perceived duration has been found to vary with processing time. Gol'dburt (1961) conducted a backward masking experiment in which he varied both the duration of the target and the intertone interval. There were three related findings of interest. First, Gol'dburt found that the presence of the mask decreased the perceived duration of the target. Second, this effect was greatest with short targets, and decreased as target duration was lengthened. Finally, the effect was found to decrease with increases in the time between the target and the mask.

The Efron (1970a, b, c) and Gol'dburt (1962) results suggest a fundamental difference between perception of duration and perception of other attributes of the auditory stimulus. That is, the percept of duration appears to vary consistently with temporal parameters, increasing with increases in stimulus duration and/or processing time. Given this potential difference, the current research attempted to analyze the temporal course of perceived auditory duration. A paradigm-backward recognition masking-was employed which is designed to explicitly tap changes in perception of a stimulus over time. The central result in this paradigm is that a second sound can disrupt perception of an earlier sound if the second sound is presented within about $1 / 4 \mathrm{sec}$ of the onset of the first sound. Backward recognition masking has been found for the perception of pitch (Hawkins \& Presson, in press; Hawkins, Thomas, Presson, Cozic, \& Brookmire, 1974; Massaro, 1970a, 1975a), timbre (Massaro, 1972b), loudness (Moore \& Massaro, 1973), vowels and consonant-vowel syllables (Massaro, 1974; Wolf, 1976), and sound lateralization (Massaro, Cohen, \& Idson, 1976). The results have been interpreted in the framework of a model that assumes that perception of a stimulus occurs over time (Massaro, 1972a, 1975b). A sound is initially stored in a preperceptual auditory store, having a capacity limit of a single sound. During the auditory recognition process, information in the store is read out continuously over the course of approximately $250 \mathrm{msec}$. If a second or masking sound is presented before processing of the first sound is completed, storage of the second sound will disrupt the representation of the first sound in preperceptual store and interfere with its processing. Thus, the improved performance with increases in the interval between the first and second sounds represents the extraction of successively greater amounts of information from the target, given longer processing times prior to the onset of the mask. The formalized model predicts that the discriminability of the target will increase as an exponential growth function of time $t$,

$$
\mathrm{d}^{\prime}=\alpha\left(1-\mathrm{e}^{-\theta t}\right)
$$

where $d^{\prime}$ indexes stimulus discriminability, $\alpha$ the amount of information in the stimulus which could be extracted given unlimited processing time, and $\theta$ the rate of processing of that information. Stimulus duration can be treated simply as another dimension of information about the sound in preperceptual store. As a result, it should take time to be perceived, since information concerning duration must be read out of preperceptual store by the recognition process.

The present research adapted a backward recognition masking task in order to explore the manner in which duration is perceived. On each trial, a $700-\mathrm{Hz}$ target tone having one of two alternative durations was presented. The target tone was followed, after a variable silent intertone interval, by a $700-\mathrm{Hz}$ masking tone which could assume one of three possible durations, symmetrical about the target durations. In order to avoid the possible use of overall loudness as a cue to duration, the intensities of the target and mask were chosen randomly on each trial within a 6-dB range. The subject's task was to determine which of the two target durations was presented on each trial.

This study should be informative with respect to several issues. First, it should illuminate the temporal course of perceived duration. To the extent that the duration percept is changing over time, performance should vary in the backward recognition masking task. Second, it will allow comparison with perception of other dimensions of sound, as measured by the same paradigm, making it possible to determine to what extent the argument that there is something unique about perceived duration is justified. If backward masking comparable to that found along other dimensions is obtained in this task, it would suggest that the uniqueness hypothesis is incorrect. Simultaneously, such a finding would provide an explanation for the perception of duration within the framework of Massaro's model (1975b). In contrast, if the data do not conform to that obtained in backward masking of other stimulus dimensions, 
the nature of the discrepancies might suggest how processing duration differs from processing other dimensions.

\section{EXPERIMENT 1}

\section{Method}

Subjects. The subjects were nine University of Wisconsin undergraduates who received extra credit in an introductory psychology course for their participation in the experiment.

Apparatus and stimuli. Four subjects could be tested simultaneously in separate sound-insulated rooms. All experimental events were controlled by a PDP-8L computer. The tonal stimuli were generated as sine waves by a digitally controlled oscillator (Wavetek Model 155) and were presented binaurally over matched headphones (Grason-Stadler Model TDH-49). The tones were turned on and off at the zero crossing and had essentially instantaneous rise times.

Two target tones were employed in the study, having durations of $50 \mathrm{msec}$ (short) and $90 \mathrm{msec}$ (long). For two subjects, this $40-\mathrm{msec}$ separation between test tones yielded performance at a level of approximately $92 \%$ correct on the practice day. In order to avoid ceiling effects, durations of 55 and $85 \mathrm{msec}$ were employed on the 4 subsequent experimental days for these two subjects only. Three masking tone durations, symmetrical around the durations of the target tones, were used-30 msec (short mask), 70 msec (mid mask), and $110 \mathrm{msec}$ (long mask). For the two highly accurate subjects, the masking tone durations were 40,70 , and $100 \mathrm{msec}$. All tones had a frequency of $700 \mathrm{~Hz}$. The intensities of the test and masking tones were sampled randomly and independently on each trial from a set of seven possible intensities: 75 to $81 \mathrm{~dB}$ SPL, in 1-dB steps.

Procedure. The experiment was conducted on 5 consecutive days. Each day was divided into two 20 -min sessions, separated by a 10-min rest break. Day 1 was considered to be practice. On the first session of that day, the subjects received 320 learning trials. On each of these trials, the subjects identified a single test tone as either long or short. Presentation of the two test tones was random and each tone was programmed to occur with equal probability. During a 1.5-sec response interval, the subjects pressed one of two buttons labeled $S$ and $L$ to indicate a short or long response. Following the response interval, feedback was provided by a 500 -msec presentation of the letter associated with the tone on a visual display of light-emitting diodes (Monsanto Model MDA-III). The intertrial interval was $1 \mathrm{sec}$.

Session 2 of Day 1 and both sessions of the 4 subsequent experimental days consisted of $\mathbf{3 2 0}$ test trials. All of the second session of Day 1 and the first 20 trials of the eight subsequent experimental sessions were treated as practice, though the subjects were not informed of this. On $7 / 8$ of the trials one of the two test tones was followed, after a variable silent intertone interval (ITI) of 5, 25, 45, 105, 165, 205, or $255 \mathrm{msec}$, by one of the three possible masking tones. On $1 / 8$ of the trials, no masking tone was presented. The response, feedback, and intertrial intervals were the same as in the practice session. All 48 experimental conditions $(2$ target durations $\times 3$ masker durations $\times 7$ ITIs plus the no mask condition) were completely random and were programmed to occur with equal probability within a session. The masking tone duration was a dummy variable under the no-mask condition.

\section{Results}

Response frequencies were pooled over the 4 experimental days. The percentage of correct judgments was computed for each subject at each target duration by masking tone duration by ITI. Two separate analyses of variance were performed on these percentages. In one, the no-mask was treated as an ITI of $\infty$ and entered the analysis as a level of the ITI factor, yielding eight levels on this factor. In the other analysis, the no-mask was excluded and the ITI factor consisted of seven levels corresponding to the seven ITIs. This second analysis was conducted so as to allow a direct evaluation of the effects of masking tone duration, eliminating those trials on which no masking tone was presented. All of the results given below which involve the masking tone duration were drawn from this latter analysis excluding the no-mask trials. All other results were taken from the analysis in which the no-mask was included.

The top panel of Figure 1 presents the average percentage of correct identifications of the target durations, as a function of the silent ITI and the duration of the masking tone. The duration of the masking tone had no effect on performance. As can be seen from Figure 1, all three masking tones produced virtually identical performance. This observation is supported by the nonsignificant effects for both the duration of the mask $(F<1)$ and the masking tone by ITI interaction.

Performance improved as a negatively accelerated function of the ITI, asymptoting at an interval of $165 \mathrm{msec}$. Performance on no-mask trials was only slightly better (approximately $1.6 \%$ ) than that found at intervals of $165 \mathrm{msec}$ or greater. This conclusion was supported by the highly significant effect of the ITI, $F(7,56)=23.29, p<.001$.

The top panel of Figure 2 presents the percentage of correct identifications of each of the two target durations as a function of the duration of the silent ITI. Quite different masking functions resulted for the two target tones. The long target produced a typical masking function: performance improved monotonically from a $25-\mathrm{msec}$ to a $255-\mathrm{msec}$ ITI. It can be seen that the long target was identified more accurately when the onset of the mask occurred $5 \mathrm{msec}$ after the offset of the target than with a 25-msec separation, an advantage of approximately $7 \%$. This departure from a strictly monotonic function is not uncommon in work in a backward recognition masking paradigm, and has been taken to reflect a period of integration of the information contained in the target with that of the mask (Massaro et al., 1976). However, in contrast to the typical findings in this type of research, performance asymptoted for the long target at a level approximately $14 \%$ higher than that produced by the nomask condition.

Performance on the short target tone was quite different from that usually found in backward recognition masking studies. Performance was close to that on the long target at the 5-msec ITI and then improved about $15 \%$ at the 25 - and $45-\mathrm{msec}$ intervals. Between 105 and $255 \mathrm{msec}$, performance remained at a level of performance approximately 


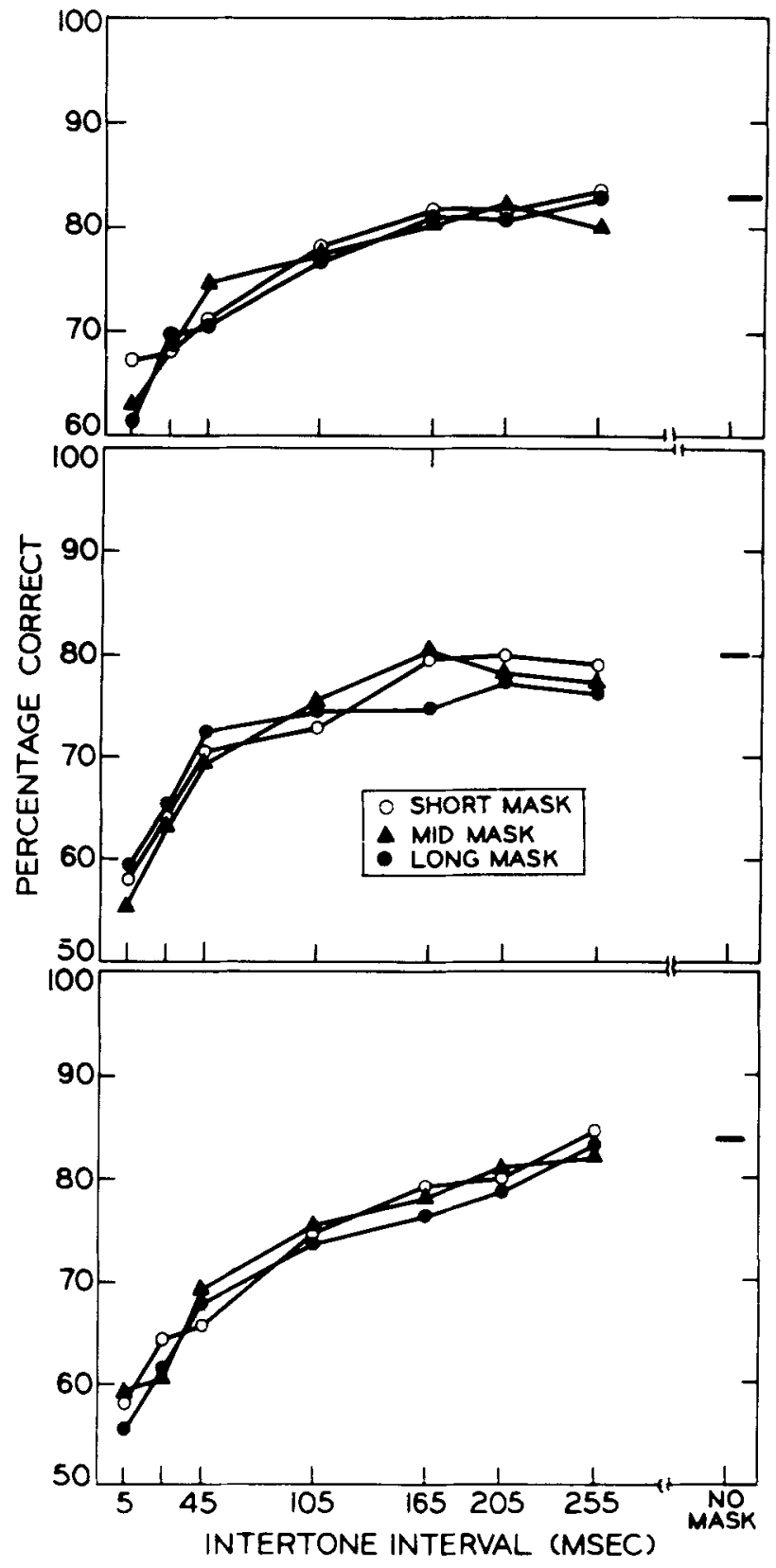

Figure 1. Percentage of correct identifications of the duration of the target tone as a function of both the duration of the masking tone and the intertone interval. The top panel represents Experiment 1, the middle panel Experiment 2, and the bottom panel Experiment 4.

$17 \%$ lower than that for the short target on no-mask trials. Statistical support for these observations is found in that while the main effect of target duration was nonsignificant, the target duration by intertone interval interaction was highly significant, $\mathrm{F}(7,56)=5.48, \mathrm{p}<.001$.

Essentially the same effects were found in the data for all nine subjects. Data from two subjects (C.B. and M.W.) are presented individually in the top panel of Figure 3.
Figure 4 presents the percentage of correct identifications of the two target tones as a function of the duration of the masking tone, pooled across ITIs. The no-mask trials were eliminated from this analysis. Identification performance is critically dependent on the relative durations of the test and masking tones. The long masking tone duration yielded poor performance on the short target and good performance on the long target, while the shortduration mask produced the opposite results. Under

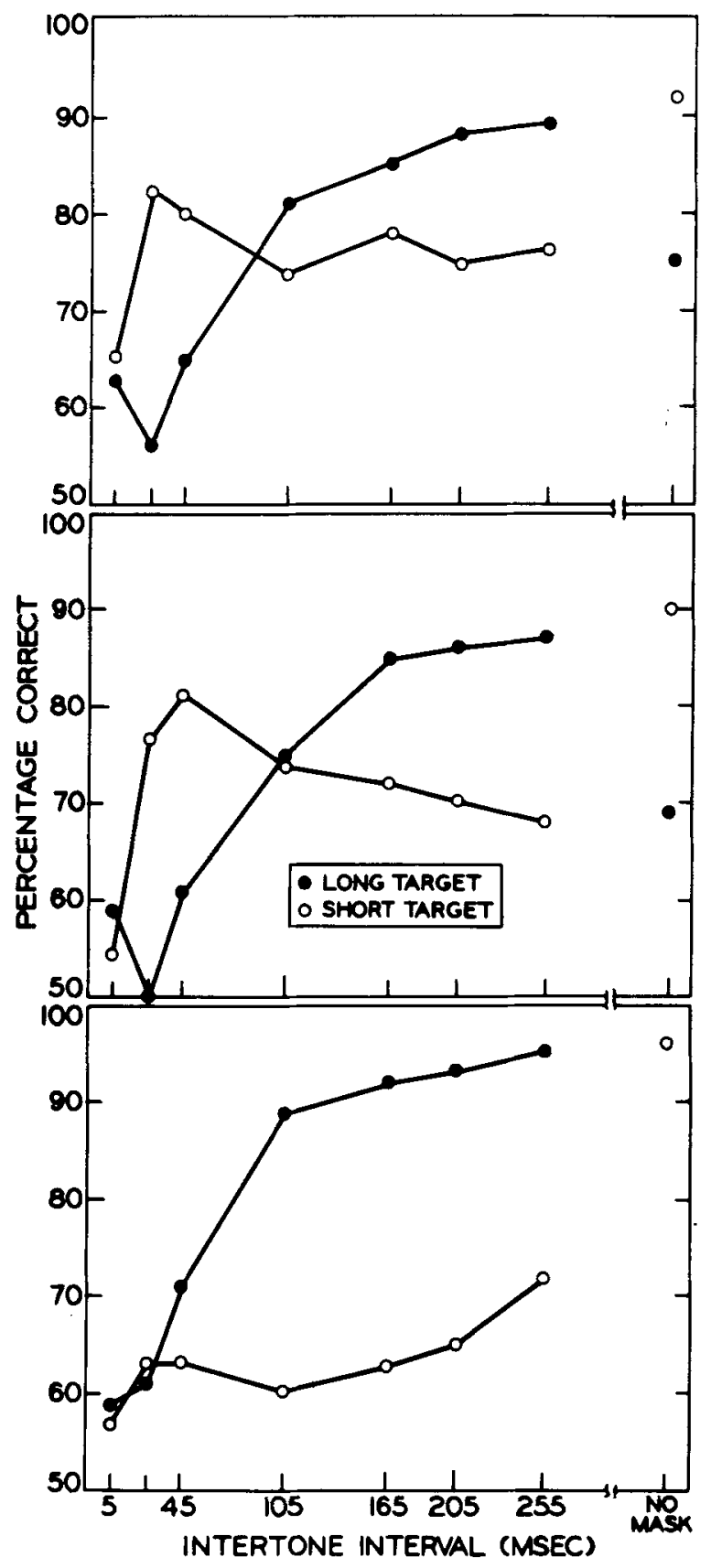

Figure 2. Percentage of correct identifications of each of the two target tones, as a function of the intertone interval. The top panel gives the results for Experiment 1, the middle panel for Experiment 2, and the bottom panel for Experiment 4. 


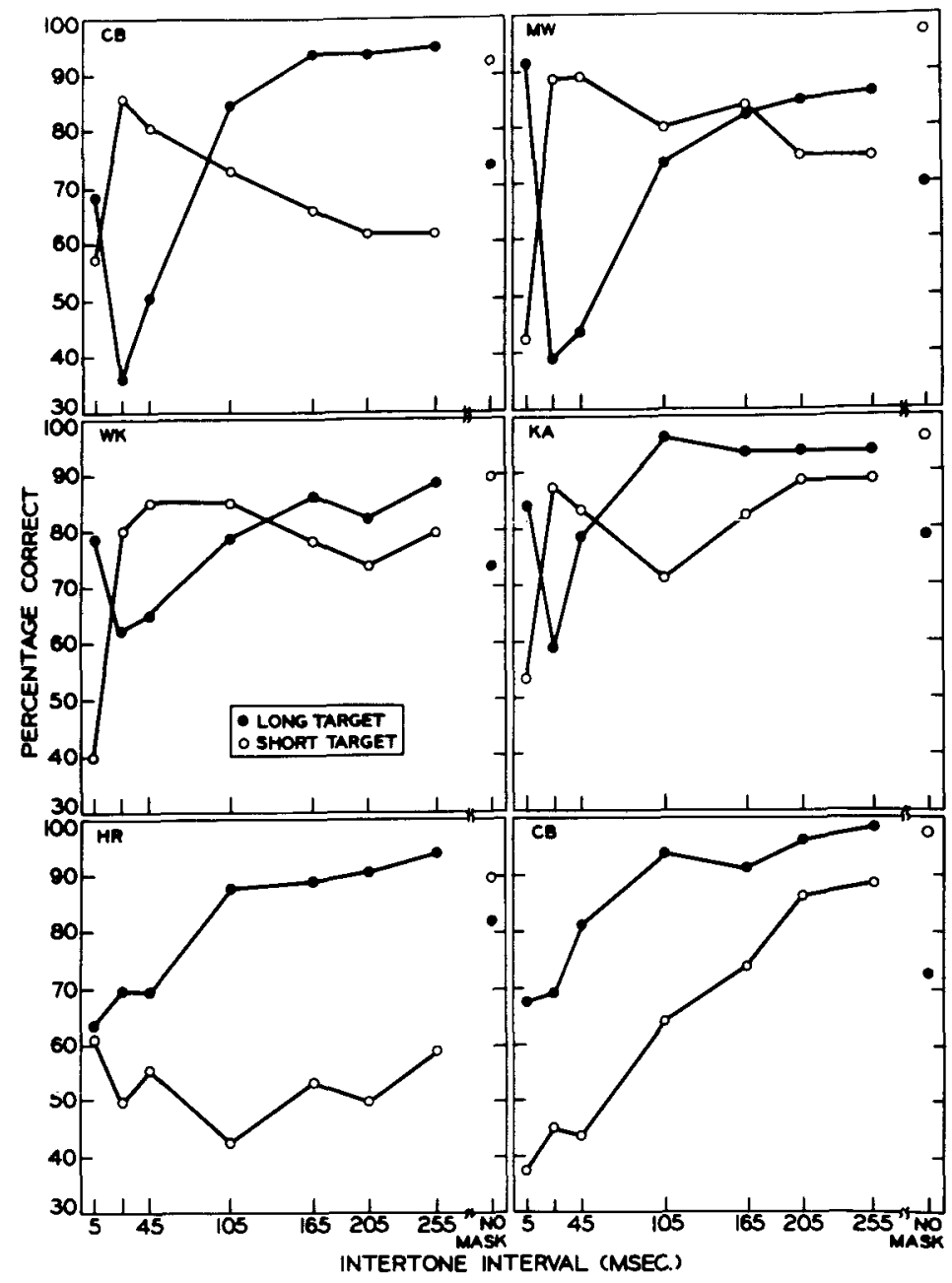

Figure 3. Percentage of correct identifications of each of the two target tones, as a function of the intertone interval. The top panel shows the data for two subjects in Experiment 1, the middle panel for Experiment 2, and the bottom panel for Experiment 4.

Figure 4. The percentage of correct identifications of the duration of each of the two target tones, as a function of the duration of the masking tones. From left to right, the three panels give results for Experiment 1,2 , and 4 , respectively. The no-mask trials have been eliminated.
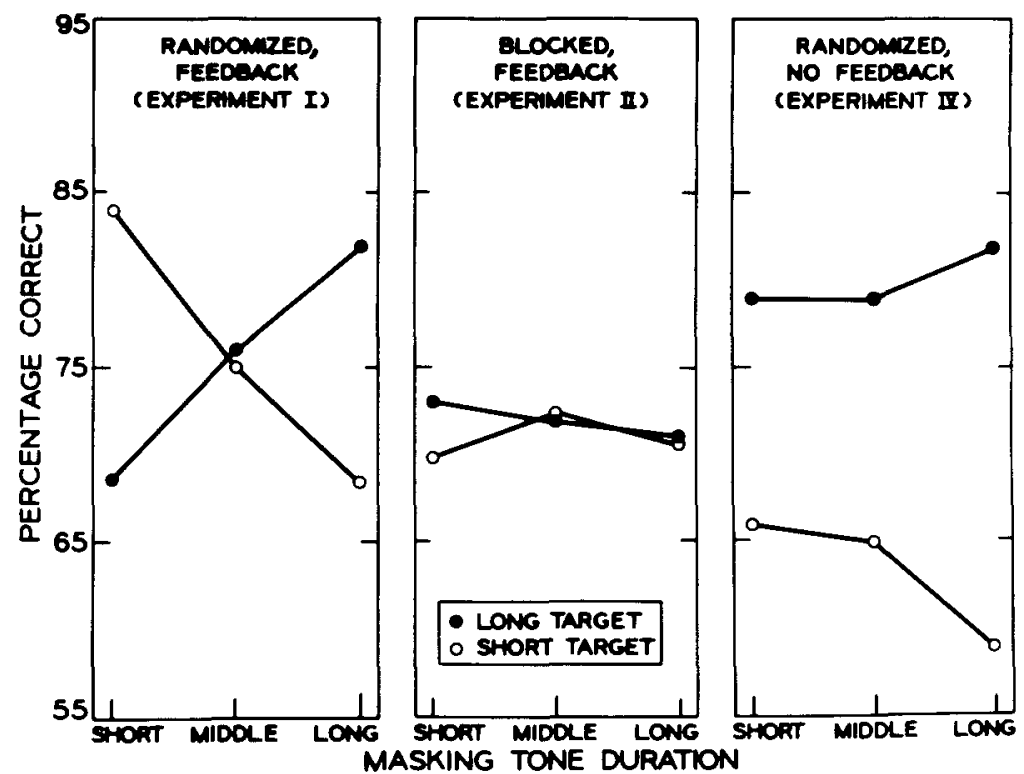
the mid-duration mask, performance on the two targets is virtually identical. These observations were supported by the analysis, which revealed a highly significant target by mask duration interaction, $F(2,16)=22.48, p<.001$.

The top panel of Figure 5 presents the percentage of correct identifications of the long and short target tones, as a function of both the duration of the masking tone and the ITI. The effect of the target duration by mask duration interaction is relatively consistent across all ITIs. Although the long and short targets produced very different masking functions, there is a consistent advantage in performance when the long (short) target is followed by the long (short) mask at all ITIs. However, the triple interaction of target duration, mask duration, and ITI was found to be significant, $F(12,96)=5.11$, $\mathrm{p}<.001$.

\section{Discussion}

The results are generally supportive of the model of backward recognition masking presented in the Introduction. The overall masking function conforms closely to the predictions of the model (cf.
Figure 1). Performance improved monotonically between a 5-msec ITI and asymptotic performance with an interval of $165 \mathrm{msec}$, at a level comparable to that found in the no-mask condition. Perception of duration does appear to occur over time, requiring an additional $165 \mathrm{msec}$ of silence after the target tone presentation. The longer the interval between target and mask, the longer the time available for complete processing of the target. As a result, performance improves monotonically with increases in the ITI. These results are in close consonance with previous research on backward recognition masking. Moreover, they agree with our interpretation of the finding of poorer discriminability of short durations in a two-interval forced-choice task with short ISIs (Carbotte, 1973; Small \& Campbell, 1962; Massaro \& Idson, Note 1).

However, there are three principal results that might be considered to be at variance with both the model and previous research utilizing a backward masking paradigm. First, performance with the short target did not improve consistently with increases in the duration of the silent ITI. The second problematic result concerns asymptotic performance on

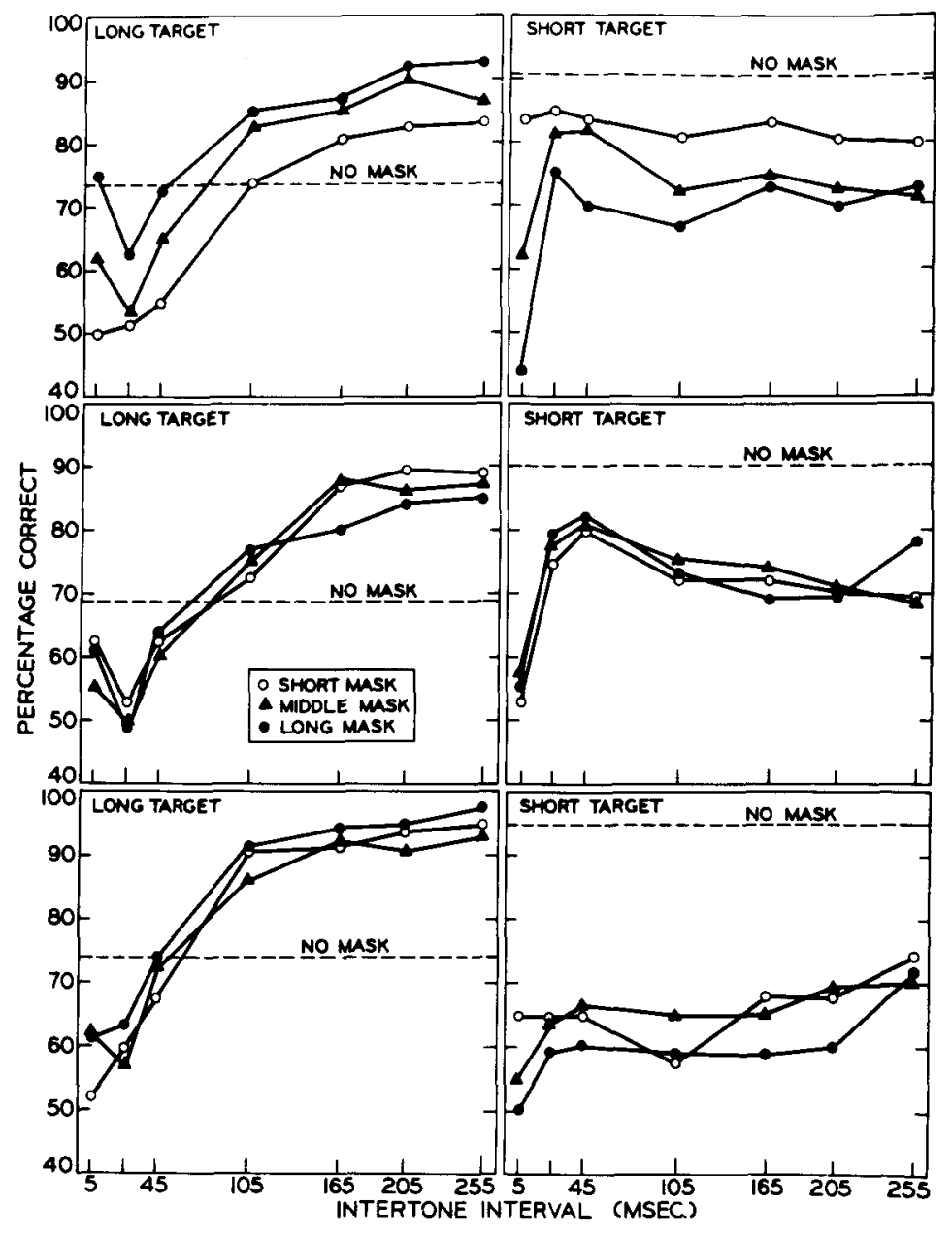

Figure 5. The left-hand panel presents the percentage of correct identifications of the long target, as a function of both the duration of the masking lone and the intertone interval. The right-hand panel shows the same data for the short target. The top panel represents Experiment 1, the middle panel Experiment 2, and the bottom panel Experiment 4. 
masking trials relative to the no-mask trials for the long and short targets considered separately. Performance on no-mask trials with the short target was approximately $92 \%$ correct, while performance for the long target on no-mask trials was at only $76 \%$ correct. Third, subjects tended to identify the target duration as long (short) when the duration of the mask was long (short). While a similar result has been found previously in backward masking of sound lateralization (Massaro et al., 1976), it does not readily conform to the model.

In terms of the theory of signal detectability (Green \& Swets, 1966), the results outlined in the preceding paragraph would be taken to represent changes in the observer's decision criteiron. However, either perceptual changes or criterion changes could account for the results. A perceptual explanation would suggest that the subjects' percept of target duration is actually changing as a function of the experimental condition. The percept may change in a given direction without necessarily affecting average percentage correct, the sensitivity measure in the experiment. Alternatively, a decision interpretation would argue that the percept remains constant, but the experimental manipulations are causing a shift in the subject's criterion for making the alternative responses.

On the basis of Experiment 1, it is not possible to distinguish between the perceptual and decision explanations for the problematic results cited above. Largely, this is due to the fact that the two interpretations can predict the same results on the basis of quite different underlying mechanisms. To illustrate this point, consider the target-mask interaction found in Experiment 1. A perceptual interpretation can be formulated in terms of a perceptual integration (Hawkins et al., 1974; Hawkins \& Presson, in press). Information from both the target and mask contributes to the perceptual experience of the target sounds. A short target integrated with a short mask will have a shorter perceived duration than a short target integrated with a mid or a long mask. As a result, the short target will be correctly identified as short more often when followed by a short mask than when followed by a mid mask or a long mask. The inverse is of course true for the long target. The long target would be most likely identified as long if it was followed by the long mask. The integration process might be operative only at short ITIs since the test and mask would not be expected to be integrated at sufficiently long ITIs (Cantor \& Thomas, 1976).

In contrast, the duration of the mask may simply alter the observer's criterion for a particular decision. Given that there is noise in the sensory system, the long and short targets can be envisioned as generating two overlapping normal distributions of perceived duration. The response is determined by place- ment of a criterion along the dimension of perceived duration. If the perceived duration of the target exceeds the criterion value for responding long, a response of long will be made; otherwise the subject will call the target short. Presentation of a masking tone may push the criterion in the direction opposite to the duration of the mask. For example, a long mask will displace the criterion further into the short distribution, causing an increased number of tones to exceed the criterion for long. This will have the effect of increasing accuracy on the long target and decreasing accuracy on the short target. Similarly, a short mask will push the criterion further into the long distribution, causing fewer tones to exceed criterion, which will decrease accuracy on the long target and increase it on the short target. Consequently, the perceptual and decision interpretations predict the same results, based upon different theoretical mechanisms. Similar contrasting interpretations can be offered for the effects of the individual target tones and the nomask condition.

As an initial hypothesis, however, a perceptual interpretation of the results appeared more promising. A perceptual interpretation would be more closely consonant with the Gol'dburt (1961) finding that the perceived duration of a short tone is shortened by a second tone to the extent the second tone occurs near the offset of the first tone. More important, in marked contrast to most backward recognition masking studies, our subjects reported that the feedback they received did not conform to their perceptual experience. Sometimes the short target sounded long, and vice versa. Furthermore, the subjects reported having heard far more than two target durations in the study. Consequently, though we are aware that a decision-oriented explanation for our results is possible, we have chosen to attempt to develop a perceptual explanation for them.

The perceptual interpretation would argue that the subjective duration of the target was changing with the ITI and the duration of the mask, a result directly comparable with Gol'dburt's (1961). This assumes that the subjective duration of these targets was not isomorphic to their objective durations. In this sense, the results are quite comparable with Efron's (1970a, b, c) finding that short target durations will be overestimated. However, Efron's data indicate that targets as short as those used in Experiment 1 should both be overestimated to have subjective durations of approximately $130 \mathrm{msec}$. Though the effects which the mask should have on such overestimates are not clear, at least on no-mask trials this implies that both targets should have approximately equal subjective durations. As the long and short targets were discriminated with much better than chance accuracy on no-mask trials, this was 
clearly not the case. It would appear that far more precise discriminations of short durations are possible than Efron had assumed, casting some doubt upon the generality of his finding that all stimuli shorter than $130 \mathrm{msec}$ are perceived as having durations of roughly $130 \mathrm{msec}$.

The introspective reports given by our subjects can be interpreted with reference to the Gol'dburt (1961) results. Recall that Gol'dburt found perceived target duration to increase with increases both in actual target duration and available processing time. This suggests that the perceived duration of the target tones in Experiment 1 may have been a function both of actual target duration and the following ITI. Moreover, though Gol'dburt did not specifically investigate this possibility, it seems reasonable to suggest that masking tone duration may also contribute to the perceived duration of the target tone. In the Hawkins model (Hawkins et al., 1974; Hawkins \& Presson, in press), for example, the durations of the target and mask would be integrated to form the final percept. Phenomenologically, Experiment 1 can be seen as containing a continuum of perceived target durations. Under such a conceptualization, the subjects' task would be to map this continuum of perceived durations into two response alternatives. It is conceivable that the interactions observed in Experiment 1 result from a strategy used by the subjects to organize a set of stimuli in terms of a binary response choice.

Experiments 2 and 3 were designed to provide more direct evidence on this issue by holding either the duration of the masking tone or the ITI constant within a block of trials. The logic of this manipulation is straightforward. Given that the target by mask by ITI interaction is creating a continuum of perceived durations, presenting only a single level of one of these variables should eliminate the influence of that variable. For example, blocking the masking tone duration should eliminate the influence of this variable. Consider the differential effects which blocking and randomizing the mask should have upon the target by mask interaction. At all ITIs, the short mask will have the effect of decreasing the perceived duration of the target tones relative to the long mask. The subject should, therefore, categorize the target tone as short more often when it is followed by a short mask than when it is followed by a long mask. When the duration of the masking tone is randomized within a block of trials, the subject can categorize the target in the direction of the mask and still maintain equal response probabilities, as the disproportionately large number of short responses with the short mask will be compensated for by a disproportionately large number of long responses with the long mask. This will produce the target by mask interaction seen in Experiment 1 and equivalent overall performance on the two targets. However, when the duration of the mask is blocked, the subject can only maintain equal response probabilities within a trial block by adjusting his/her criterion according to the particular masking tone duration presented in that block. In this case, the short mask should not yield a greater number of short responses than the long mask, eliminating the target by mask interaction.

\section{EXPERIMENT 2}

Experiment 2 provides a direct replication of Experiment 1, with the exception that the duration of the masking tone was blocked across experimental sessions. Consider the effect that this manipulation should have if the theoretical framework offered above has validity. At any given ITI, each masking tone is interacting with each target tone, yielding six subjective durations. The subjects' task is to establish a response criterion which will distinguish between those subjective durations which should be called long and those which should be called short. Consequently, to the extent that the target and mask are both short (long), the perceived duration will be correctly categorized, while to the extent that the target is short (long) and the mask is long (short) the categorization will be incorrect. As can be seen from Figure 4, this is the general form of the interaction between target and mask which was observed in the first experiment. However, when only a single mask duration occurs within a block of trials, only two perceived durations-one long and one shortwill occur at any given ITI. Given that the feedback informs the subject that the long and short test tones are equally likely, the subject will set his/her criterion to respond long and short with equal probability regardless of the duration of the masking tone. If this argument is valid, blocking the duration of the masking tone should eliminate the target by mask interaction completely. The blocking manipulation should, however, have no effect on the target by ITI interaction since responding short to the short perceived durations experienced at short ITIs can be compensated by long responses to the long perceived durations at long ITIs.

\section{Method}

Subjects. The subjects were six University of Wisconsin undergraduates who received academic credit for their participation. The subjects were tested in two groups of three subjects each.

Procedure. The procedure for Experiment 2 was identical to that in Experiment 1, with two exceptions. First, the duration of the masking tone was now blocked across experimental sessions, only a single masking tone duration being employed within a session. Subjects were given two practice sessions, followed by one experimental session on Day 1. Days 2-5 had two experimental sessions per day, yielding a total of nine experimental sessions. A given masking tone duration was presented once in each block of three sessions, counterbalancing the order of presentation over groups and sessions. The second change was 
that the durations of the target tones were altered to avoid ceiling effects which would obscure the results. The long target now assumed a duration of $80 \mathrm{msec}$ and the short target the duration of $60 \mathrm{msec}$. The masking tone durations assumed values of 50 , 70 , and $90 \mathrm{msec}$. It should be noted that the relationship between the target and the masking tone durations is the same in Experiments 1 and 2 . In both cases, the middle mask is halfway between the targets and the short (long) mask differs from the short (long) target by $1 / 2 \Delta t$ where $\Delta t$ is the difference between the long and short targets.

\section{Results}

An analysis of variance was conducted with subjects, replications $(1,2$, and 3$)$, targets, masks, and ITIs as factors. The main effect of replications was not significant, the largest difference in performancebetween replications 1 and 2 -being only $6 \%$. All interactions involving replications as a factor were also nonsignificant. These nonsignificant results indicate that no appreciable learning was occurring across sessions, which might introduce irrelevant variation due to the blocking manipulation. Consequently, to increase the reliability of the individual subjects' scores, the response frequencies were pooled over the three replications and reanalyzed. Once the data had been pooled across replications, two analyses of variance were again performed, as in Experiment 1.

The middle panel of Figure 4 presents the percentage of correct identifications of the long and short targets, as a function of the duration of the masking tone. It is apparent that blocking the duration of the mask eliminated the target by mask interaction entirely, all three masking tones producing the same effects. The middle panel of Figure 5 shows that masking tone duration failed to have an effect at all ITIs. These conclusions are confirmed by the absence of a significant target by mask or target by mask by ITI interaction $(F<1)$.

The middle panel of Figure 1 presents the average percentage of correct identifications as a function of the duration of the silent ITI and the masking tone duration. As in Experiment 1, no differential effects were found for the three masking tone durations, as indicated by the absence of both a main effect of masking tone duration $(F<1)$ and a mask by ITI interaction $(F<1)$. For all three masks, performance is a negatively accelerated function of the duration of the silent ITI, asymptoting at $165 \mathrm{msec}$, at a level of performance comparable to that found in the no-mask condition, $\mathrm{F}(7,35)=12.69, \mathrm{p}<.001$.

The middle panel of Figure 2 shows the percentage of correct identifications of each of the target tones as a function of the duration of the silent ITI. The Target Duration by ITI interaction found in Experiment 1 was replicated exactly, $F(7,35)=4.21$, $\mathrm{p}<.005$. Performance on the long target was quite similar to that found in Experiment 1, a monotonically increasing function between a 25 - and 255 msec ITI. The curve for the short target was also similar to that found in Experiment 1. Performance improved rapidly from near chance at the $5-\mathrm{msec}$ interval and was nearly $20 \%$ better than performance on the long target at intervals of 25 and $45 \mathrm{msec}$. However, performance then decreased and the short target was identified much less accurately than the long target at all intervals of $105 \mathrm{msec}$ or greater.

The same effects seen in the group data were present in the data for the individual subjects. The middle panel of Figure 3 presents representative data from two individual subjects (W.K. and K.A.).

\section{Discussion}

The results of Experiment 2 support the theoretical framework given above. Perceived duration does appear to increase with increases in actual target duration, ITI, and mask duration. Blockirg the masking tone durations does not permit the subject to respond differentially as a function of this variable and still maintain an equal proportion of short and long responses. This eliminated the target by mask interaction, but had no effect on the target by ITI interaction.

\section{EXPERIMENT 3}

The logic of Experiment 3 is identical to that for Experiment 2. Given a masking tone of fixed duration, the perceived duration of the target will be a function of both actual target duration and the following ITI. This would again create a continuum of perceived durations which the subject must partition into two responses. As the duration percept has been found to increase with increases in the ITI (Gol'dburt, 1961), a greater proportion of targets would be categorized as short with short ITIs and as long with long ITIs. This is exactly the form of the target by ITI interaction seen in Experiments 1 and 2. However, when only a single ITI is presented within a block of trials, responding short to short perceived durations at a short ITI would produce more short than long responses. Given that subjects will usually attempt to equalize the proportion of short and long responses within a block of trials with feedback and an a priori probability of .5 , the subject will adjust his criterion accordingly. The results should, therefore, show that the subject responds with equal probability at a given ITI, eliminating the target by ITI interaction. Experiment 3 thus replicates Experiment 2, except that the ITI rather than masking tone duration was now blocked across experimental sessions.

\section{Method}

Subjects. The subjects were eight University of Wisconsin undergraduates who received academic credit for their participation. The subjects were tested in two groups of four subjects each.

Procedure. The procedure for Experiment 3 was identical to that for Experiment 1, with three exceptions. First, only four 
intertone intervals were employed: $25,85,165 \mathrm{msec}$, and no mask. Second, the duration of the ITI was now held constant within an experimental session. Day 1 was considered a practice day. The subjects were tested for four short sessions of 150 trials each, one of the four ITIs being presented on each session. For both groups of subjects, the no-mask session occurred first on Day 1, to allow the subjects to learn the two target tone durations absolutely, as in the earlier studies. The presentation order of the ITIs for the subsequent three sessions of Day 1 was: Group 1, 25, 85, 165 msec; Group 2, 165, 85, $25 \mathrm{msec}$. Days 2-5 had two experimental sessions per day, yielding a total of eight experimental sessions for each of the two groups of subjects. In each session, a single ITI was employed, with order of presentation counterbalanced across the 16 experimental sessions. Third, in order to avoid ceiling effects in the data, the target tone durations were changed to 60 and $80 \mathrm{msec}$ for the short and long target tones, respectively. The masking tones assumed durations of 50,70 , and $90 \mathrm{msec}$, keeping the target-tone/masking-tone relationships comparable to those employed in the previous studies.

\section{Results}

First, an analysis of variance was carried out with subjects, replications (1 and 2), targets, masks, and ITIs as factors. The main effect of replications was nonsignificant $(F<1)$, performance on the two

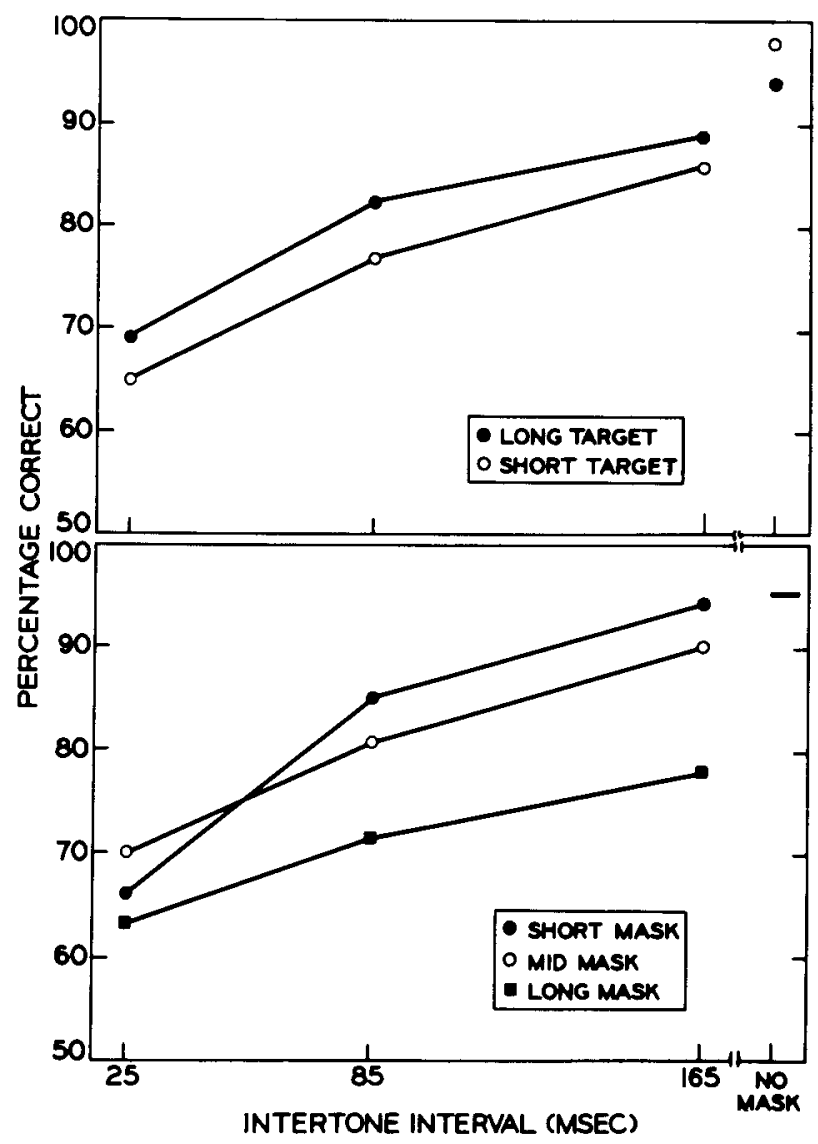

Figure 6. Top panel: Percentage of correct identifications of the long and short target tones as a function of the intertone interval. Bottom panel: Average percentage of correct identifications of the target tone as a function of the masking tone duration and the intertone interval. Experiment 3.

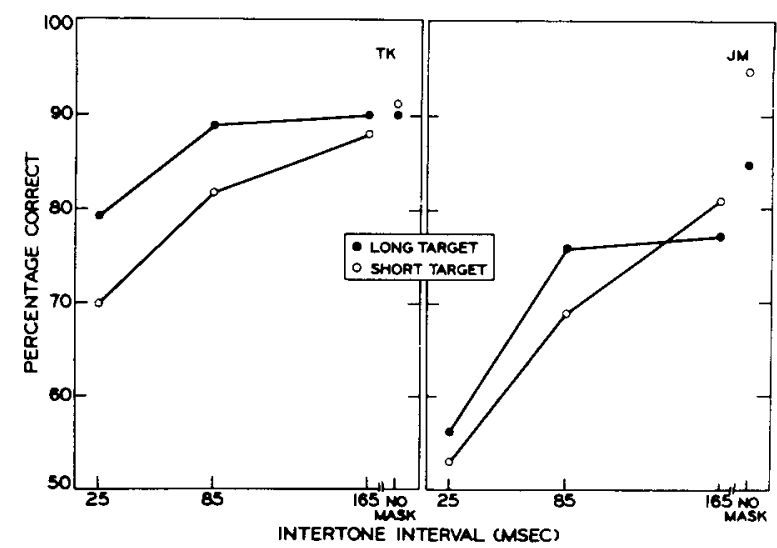

Figure 7. Percentage of correct identifications of each of the two target tones, as a function of the intertone interval. Two observers in Experiment 3.

replications differing by less than $3 \%$. All interactions involving replications as a term were also nonsignificant. These results indicate that learning was not a significant factor during the 4 days of the experiment and, therefore, that blocking the ITI should not have introduced extraneous differences between the conditions. Consequently, to increase the reliability of the individual subject scores, the response frequencies were pooled over the two replications and reanalyzed as in the previous experiments.

The top panel of Figure 6 presents the percentage of correct identifications of the two target tones, as a function of the duration of the silent ITI. In marked contrast to the results of the previous studies, the two target tones produced qualitatively similar masking functions. Performance improved with increases in the ITI leveling off about $8 \%$ lower at the 165-msec ITI than in the no-mask condition. Identification of the long target was $4 \%$ better on no-mask than on the 165 -msec ITI trials, while identification of the short target was $11 \%$ better. The interaction of target duration and ITI was not significant.

The eight individual subjects produced data similar to that seen in the group results. The data for two subjects (T.K. and J.M.) are presented separately in Figure 7.

The bottom panel of Figure 6 presents the percentage of correct identifications as a function of the silent ITI and the masking tone duration. In contrast to the earlier studies, the three masking tone durations yielded somewhat different results. For all three masks, performance was a negatively accelerated monotonic function of the ITIs. However, the long mask produced substantially greater decrements in performance than did the other two masking tones. These results were confirmed by the significant main effects of the masking tone duration, $F(2,14)=5.18, p<.025$, and the ITI, 


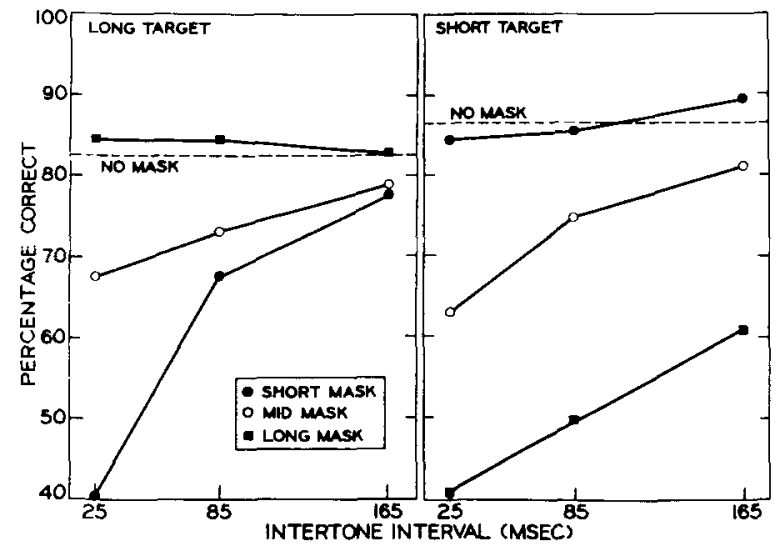

Figure 8. Percentage of correct identifications of each of the two target tones, as a function of the intertone interval and the masking tone duration in Experiment 3.

$F(3,21)=32.1, p<.001$. The interaction of these variables was nonsignificant.

The masking tone duration by target tone duration interaction replicated the findings of Experiment 2. Identification of the long target was $28 \%$ better when it was followed by the long rather than the short mask, while the reverse relationship obtained for the short target tone. Once again, identification of the two target tones was essentially equivalent under the mid-duration mask. These results are supported by the highly significant interaction of target and mask found in the analysis, $F(2,14)=55.43$, $\mathrm{p}<.001$. Figure 8 shows the significant interaction of target, mask, and ITI, $F(6,42)=18.49, \mathrm{p}<.001$.

\section{Discussion}

Blocking the ITI across experimental sessions completely eliminated the target duration by ITI interaction seen in the earlier experiments. This result supports the idea that perceived duration increases over time, the subjective duration of a stimulus being a function of both the actual duration of the stimulus and the following ITI. It should be pointed out that the increase in perceived duration with increases in ITI does not necessarily mean that the probability of being correct on the short target should decrease with increases in ITI. This follows from the fact that increases in ITI also increase the difference in the perceived durations of the short and long targets so that the general increase in overall accuracy can offset the expected increase in errors on the short target resulting from the increase in perceived duration with increases in ITI.

\section{EXPERIMENT 4}

The results of Experiments 2 and 3 clearly support the theoretical interpretation suggested for the interactions found in Experiment 1. Perceived target duration does seem to increase with increases in actual target duration, mask duration, and ITI, yielding a set of perceived durations for the two actual target durations. The target by mask and target by ITI interactions seen in Experiment 1 appear to result from a decision rule utilized by the subjects in mapping this set of perceived durations into two responses.

However, this proposed strategy assumes that the subject attempts to utilize an optimal criterion in categorizing the perceived target durations. In all three of the preceding experiments, the subjects were instructed that the two target tones occurred equally often and received feedback as to which tone was presented on each trial. In this situation, an optimal criterion would ensure that the probability of making a long or short response was .5. In fact, in all three studies, the probability of a long response never differed from that for a short response by more than $3 \%$. Consequently, if we are correct in assuming that the critical interactions result from a decision strategy, then not inducing the subjects to equalize their response probabilities should change the nature of the observed interactions. Eliminating feedback might allow the subject to provide a more direct index of his perceptual experience of the target tone under the various experimental conditions. Experiment 4 was designed to evaluate this suggestion, by eliminating feedback within the experiment.

\section{Method}

Subjects. The subjects were seven University of Wisconsin undergraduates who received credit for their participation towards an introductory psychology course.

Procedure. The procedure and data analysis were identical to that of Experiment 1, with one exception. On Day 1, feedback was provided during the learning trials with no masking tones in Session 1, as in Experiments 1 and 2. On Session 2 of Day 1 and on the four subsequent experimental days, the subjects did not receive feedback as to the correct response. Instead, an asterisk (*) appeared over the light-emitting diodes, at the point at which the feedback had been given. This asterisk signified the end of the response interval. The test and masking tones were the same as those used in Experiment 1.

\section{Results}

The bottom panel of Figure 1 presents the average percentage of correct identifications of the duration of the target tone as a function of the silent ITI. As can be seen in the figure, the three masking tones again produced identical effects and performance was a negatively accelerated function of the ITI, $F(7,42)=20.2, p<.001$. Both the main effect of masking tone duration $(F<1)$ and the mask by ITI interaction $(F<1)$ were nonsignificant in the analysis. Unlike the earlier experiments, the curve did not asymptote at $165 \mathrm{msec}$, and performance improved about $5 \%$ between the 165 - and 255 -msec intervals.

The bottom panel of Figure 2 presents the per- 
centage of correct identifications of each of the two target tones, as a function of the duration of the silent ITI. The two target durations again produced quite different results. Identification of the long target improved as a monotonic function of the ITI. Performance on the long target on no-mask trials was approximately $22 \%$ poorer than identification of the long target at the longest ITI. Identification of the short target improved at a rate comparable to that of the long target between intervals of 5 and $25 \mathrm{msec}$, but at a much slower rate after this point. In contrast to the results of Experiments 1 and 2, performance on the short target increased by approximately $12 \%$ between intervals of 105 and $255 \mathrm{msec}$. The short target was identified $24 \%$ more accurately without a masking tone than it was at the longest processing interval with a masking tone. These results were confirmed by a significant target duration by ITI interaction, $F(7,42)=3.59, \mathrm{p}<.01$.

The seven individual subjects produced data similar to that seen in the group data. The data for two subjects (H.R. and C.B.) are presented separately in the bottom panel of Figure 3.

The right panel of Figure 4 presents the masking tone duration by target tone duration interaction. The most noticeable result is the pronounced tendency of the subjects to categorize the target as long rather than short. Furthermore, the influence of masking tone duration had a much smaller effect than it did in Experiment 1. Supporting this, the target duration by masking tone duration interaction was not significant, $F<1$. The bottom panel of Figure 5 shows that the triple interaction of target duration, masking tone duration, and ITI was not significant.

\section{Discussion}

The two target tone durations again produced differential results when feedback information was eliminated. However, the shapes of the masking functions departed somewhat from those found in Experiments 1 and 2. In contrast to the previous results, identification of the short target did not show an advantage over the long target at very early intervals. At intervals of 5 to $25 \mathrm{msec}$, the two targets were identified with equal accuracy, at a level only slightly greater than chance. At intervals of greater than $25 \mathrm{msec}$, performance improved for both targets but much more slowly for the short than for the long target. This finding of improved performance on the short target at long intervals contrasts with the finding of constant or decreasing performance at these intervals in the previous experiments. These discrepancies would appear to result from a strong bias on the part of the subjects to categorize both target tones as being long. In accord with the idea that eliminating feedback would reduce the subjects' tendency to equalize their response probabilities over the two alternatives, the long tone was identified $12 \%$ more accurately than the short tone averaged over all experimental conditions. This bias to respond long had the simultaneous effects of lowering the curve for identification of the short target and raising the curve for identification of the long target. As a result, the advantage of the short target at early intervals was eliminated and the distance between the two curves increased at the long intervals. However, despite the unequal response probabilities, the results of the no-mask trials replicated those found in Experiments 1 and 2, with a large tendency to respond short being observed.

The findings of Experiment 4 provide support for the hypothesis that the form of the interactions observed in the earlier experiments do, in fact, result from a decision rule designed to maintain an equal probability for the two responses. In Experiment 4, when feedback was eliminated, subjects showed a much greater tendency to respond long than short on the masking trials. This had the result of strongly attenuating the target by mask interaction. Though a target by ITI interaction still occurred, it primarily reflected a difference between the two targets in the rate of improvement of performance across ITIs. The marked cross-over of the two curves observed in Experiments 1 and 2 was completely eliminated. The target by mask interaction was virtually eliminated, with the exception that the long mask still resulted in more accurate identifications of the long than of the short target.

\section{EXPERIMENT 5}

Experiments 2-4 demonstrate that two of the problematic results uncovered in Experiment 1 can be accounted for in terms of the proposed explanation for perceived duration. However, the third of these results remained invariant across the manipulations employed in these studies. That is, performance on a given target failed to asymptote within $250 \mathrm{msec}$ at a level of performance comparable to that found for the same target on no-mask trials. Moreover, while performance at long masking intervals was always better on the long target, performance on no-mask trials was always better on the short target.

The purpose of the fifth experiment was to demonstrate that the observed differences in the individual targets on asymptotic mask and no-mask trials is due to whether or not the masking tone is incorporated into the duration judgment. The longest ITI in the previous experiments was $255 \mathrm{msec}$, and it is unlikely that the subject could make a response before hearing a masking tone. By increasing the duration of the ITI, however, subjects should be able to make a greater proportion of judgments before the masking tone is presented. In this case, the asymp- 
totic performance on masking trials should approach that of no-mask trials as the ITI is increased. Experiment 5 replicated Experiment 1 exactly, but included longer ITIs out to $1 \mathrm{sec}$. If the differences between the masking and no-mask trials are due to incorporating the duration of the masking tone into the duration judgment, the curves for the two targets should eventually reach the same level of performance as that observed on no-mask trials.

\section{Method}

Subjects. The seven subjects were University of Wisconsin undergraduates who received academic credit for their participation.

Procedure. The procedure and data analysis for Experiment 5 was identical to that for Experiment 1, with two exceptions. First, the ITI values were altered so as to include longer intervals: $25,85,165,225,505,755,1,005 \mathrm{msec}$ and no-mask trials. Second, in order to avoid ceiling effects at the longer intervals, the durations of the long and short targets were changed to 80 and $60 \mathrm{msec}$, respectively. The masking tones assumed values of 50,70 , and $90 \mathrm{msec}$, symmetrical around the values of the target tones.

\section{Results}

The top panel of Figure 9 presents the percentage of correct identifications of each of the target tones, as a function of the duration of the silent ITI. At intervals of $25-255 \mathrm{msec}$, the current results replicate those found in Experiments 1-3 exactly. Performance on the long target improved monotonically between a 25-msec ITI and asymptotic performance at a 255-msec interval. Asymptotic performance on the long target was about $5 \%$ better than on no-mask trials for that target. Performance on the short target was approximately $25 \%$ better than that on the long target at a $25-\mathrm{msec}$ ITI and then decreased, resulting in poorer performance on the short target at ITIs between 85 and $255 \mathrm{msec}$. Performance was essentially constant between ITIs of 255 and $505 \mathrm{msec}$. However, performance on the short target increased between 505 and 1,005 msec, reaching a level of accuracy equal to that found on no-mask trials, while performance on the long target decreased slightly. These results are confirmed by the significant target duration by ITI interaction, $F(7,42)=11.00$, $p<.001$, though the main effect of target tone duration was nonsignificant.

The same effects seen in the group were present in the data for the individual subjects. Data for two subjects (L.R. and G.G.) are shown individually in the two bottom panels of Figure 9.

As in Experiments 1, 2, and 4, no differential effects were found for the three masking tones. For

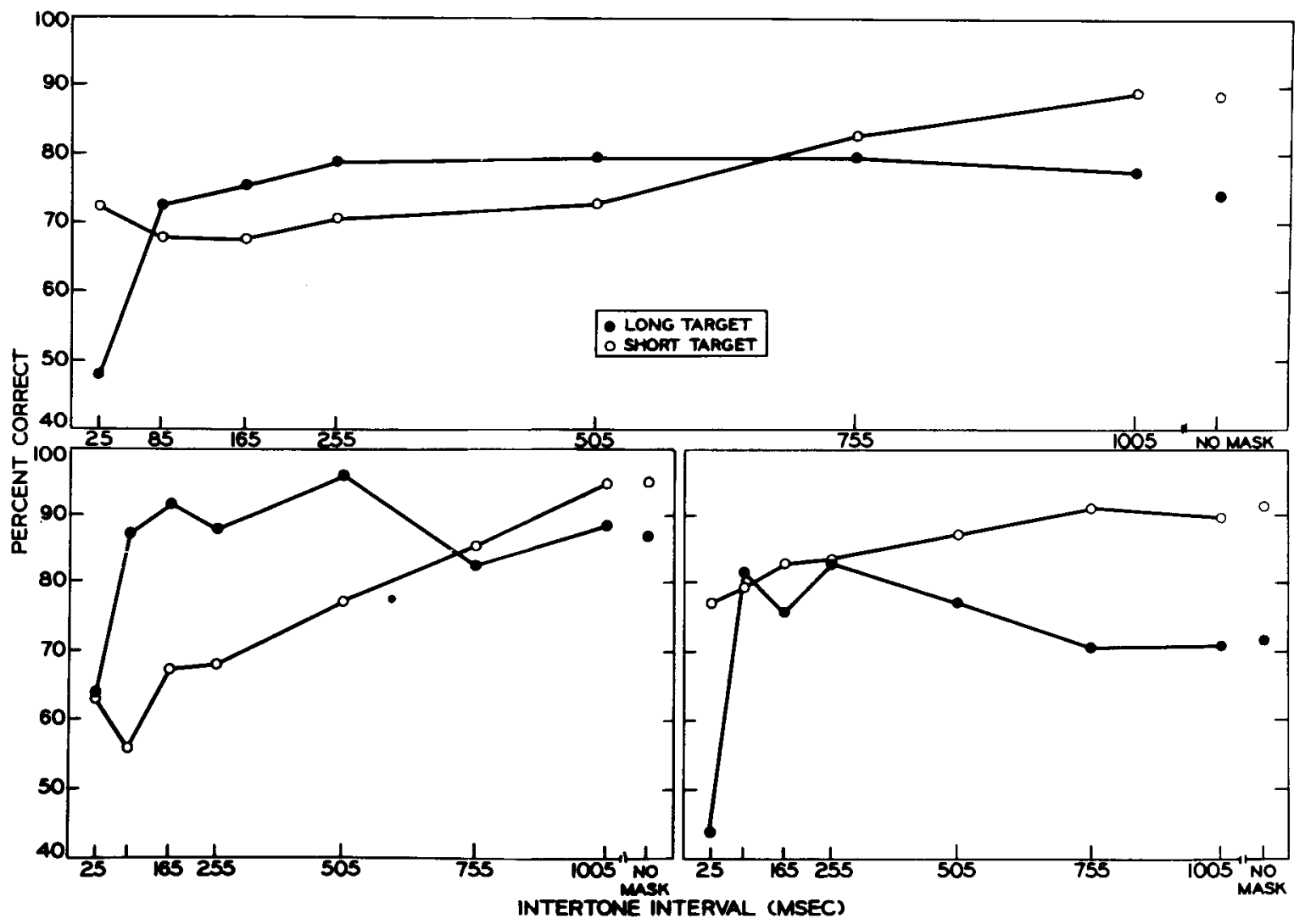

Figure 9. The percentage of correct identifications of the long and short target tones as a function of the intertone interval in Experiment 5. The top panel gives the group results, and the bottom panels give the results for two individual observers, L.R. and G.G. 
all three masks, performance was a negatively accelerated function of the duration of the silent ITI (cf. Figure 9). These observations were confirmed by the nonsignificant main effect of masking tone duration, the nonsignificant masking tone by ITI interaction, and the highly significant main effect of the ITI, $F(7,42)=21.81, p<.001$.

The target tone duration by masking tone duration interactions replicated Experiments 1, 3, and 4. Performance was dependent on the target-mask interaction, with the long (short) target being identified more accurately when followed by the long (short) mask, $F(2,12)=14.87, p<.001$. However, these effects were much smaller than those seen in the earlier studies. This smaller interaction may be due to the decrease in the target-mask interaction seen at the longer ITIs. For the long target, the targetmask interaction was quite strong between 25 and $505 \mathrm{msec}$, but decreased greatly after this point. For the short target, however, convergence of the three masks was not seen until the longest interval of $1,005 \mathrm{msec}$. The triple interaction of target, mask, and ITI was also significant in the analysis, $F(12,72)=2.93, p<.01$.

\section{Discussion}

Experiment 5 demonstrated that with sufficiently long ITIs, performance on both targets reached a level comparable to that found on no-mask trials. Identification of the long target improved until an ITI of $255 \mathrm{msec}$, and decreased after this point. Performance on the short target, however, continued to improve out to an ITI of $1,005 \mathrm{msec}$, reaching the same level of performance as in the no-mask condition.

These results support the proposed explanation for the differences between the asymptotic masking and no-mask trials. As the ITI was increased to longer values, subjects could make a greater proportion of judgments before the masking tone was presented. Accordingly, average performance at these ITIs resulted from a mixture of both "masking" and "no-mask" trials. Increasing the ITI, therefore, had the effect of increasing performance on the short target and decreasing performance on the long target, producing a crossover of the curves for the two targets. With an ITI of $1 \mathrm{sec}$, performance on the two targets approached the same levels of performance seen on the no-mask trials.

\section{GENERAL DISCUSSION}

Before considering the results of the current set of experiments with respect to duration, a more general point needs to be made. A critical methodological assumption underlying this research is that it is the psychological rather than the physical nature of the task which must be held constant between experiments. The importance of this assumption can be seen in a comparison of Experiments 1 and 2 . Blocking the duration of the mask in Experiment 2 decreased the number of experimental conditions withih a session by $2 / 3$, greatly reducing stimulus uncertainty relative to Experiment 1 . This had the effect of decreasing task difficulty and increasing the subject's sensitivity. In order to maintain performance at the same level for the randomized and blocked masking tone studies, the difference in duration between the two targets had to be halved in the blocked experiment. This clearly altered the physical stimulus situation. However, the masking tone durations were decreased in accord with the target durations, maintaining the same relationships among stimuli in the two experiments. With this alteration, almost identical results were obtained in the two experiments, for all conditions which were not explicitly affected by blocking the mask. As a result, a clear interpretation of the effect of blocking was possible. Had the durations not been altered in Experiment 2, the psychological situation would have been different in the two studies, as the tones in Experiment 2 would have been more discriminable. Performance under these conditions would have been so good that ceiling effects would have obscured all differences among experimental conditions. To have concluded from such results that blocking the mask eliminated masking and all other effects found in Experiment 1 would clearly have been incorrect, as Experiment 2-which avoided ceiling effectsreplicated Experiment 1. It can be seen that, in the current studies, controlling the stimulus situation at the expense of the psychological situation would have resulted in ambiguous data and possibly erroneous conclusions.

The results of Experiments 1-5 indicate that discrimination between two target durations can be handled in the same general manner as discrimination of targets differing along other auditory dimensions. In accord with previous research in a backward recognition masking paradigm, discrimination of duration was found to improve over time. In terms of the general model of auditory recognition (Massaro, 1975b), this can be interpreted as representing a readout of information concerning duration from an early preperceptual memory. Presenting a masking tone prior to completion of processing of the target disrupts target information and terminates any further discrimination.

However, several results clearly did not replicate earlier work, most particularly the findings for the individual target tones. The suggestion was made above, which is supported by the results of Experiments $2-5$, that these discrepancies result from somewhat unique aspects of the perceptual experience of stimulus duration. The question of interest then concerns the nature of this perceptual experience. 
Along other auditory dimensions, discriminability increases with the intertone interval. For example, the pitch of a target tone will be perceived more accurately with increases in the intertone interval, but a $500-\mathrm{Hz}$ tone will not be consistently perceived as having the pitch of a $200-\mathrm{Hz}$ tone at a $5-\mathrm{msec}$ interval, of a $300-\mathrm{Hz}$ tone at a $100-\mathrm{msec}$ interval, and an accurate pitch at asymptotic performance. For duration, however, subjective duration varies consistently from the actual duration of the target across ITIs. It should be stressed that this change in perceived duration occurs simultaneously with changes in discriminability.

We have developed a model for perceived duration based upon Massaro's (1975b) model of auditory recognition, which encompasses the Efron (1970a, b, c) and Gol'dburt (1961) results. The model assumes that the perception of duration occurs in essentially the manner described above. At presentation, the target tone is stored in preperceptual memory. During the recognition process, the information contained in preperceptual memory is read out over time, increasing the discriminability of one target from another. However, additional assumptions are incorporated into the model to account for perceived duration. The principal assertion is that the perceived duration of the target increases over time as a negatively accelerated monotonic function. Given sufficient processing time, the perceived duration of the target will reach an asymptotic value which is directly related to its actual temporal extent. However, if a mask is presented which terminates processing of the target before this asymptotic value is reached, then the perceived duration of the target will be less than its actual temporal extent. As processing is assumed to begin with the onset of the target, the perceived duration will be a function of both the actual target duration and the intertone interval. Consequently, processing time for duration is indexed by the stimulus onset asynchrony (SOA) between the target and the mask. In addition, the mask not only decreases the perceived duration of the target by terminating processing, but is also assumed to add a constant to perceived target duration. This constant is taken to be proportional to the actual duration of the mask, the long mask adding a greater amount to the perceived duration of the target than does the short mask. Consequently, the mask serves to lengthen the perceived duration of the target, relative to the perceived duration of that same target on no-mask trials.

These two processes, the growth of the percept of target duration over time and the lengthening effect of the mask, work together to determine the final subjective duration of the target. As a result, the combination of the target, mask, and intertone interval durations will create a continuum of perceived durations. Despite the fact that only two target durations were present in the current experiments, the central implication of the assumptions described above is that a whole continuum of perceived durations existed for the subject. However, the masking paradigm did not allow the subject to directly report these perceived durations, as only two response alternatives were available. The subjects were required to map the continuum of subjective durations into two discrete response alternatives. The durations of the targets, the masks, and the intertone intervals determine the range of perceived durations in the experiment. The particular subjective duration present on a given trial would simply represent some point along this continuum. The subject's response strategy would then consist of placement of a decision criterion that would partition the continuum into values of perceived duration to be categorized as long and values to be categorized as short. The decision criterion represents the value of perceived duration which must be exceeded in order for the subject to respond long. Then, if the perceived duration on a given trial is greater than this value, the target will be called long. If the perceived duration is less than the criterion value, the target will be called short. The model assumes that a single and optimal criterion will be utilized throughout the experiment.

The model can handle the results of the current studies directly. At short intertone intervals, there will be relatively little processing time available, and both targets will have relatively short perceived durations. As a result, the perceived durations of the targets will only rarely exceed the criterion for responding long, and the target will almost always be categorized as short. This will ensure accurate identification of the short target, but quite poor identification of the long target. As the intertone interval is increased, increasing the available processing time, the perceived durations of both targets will also increase. Consequently, as the criterion is fixed, a successively greater proportion of the targets will exceed criterion and will be categorized as long. This will have the effect of simultaneously increasing accuracy on the long target and decreasing accuracy on the short target. The model thus can predict the exact form of the target duration by intertone interval interaction (cf. Figures 2, 3, and 9). The long target was identified more accurately with increases in the intertone interval, while the short target was identified most accurately at the short intervals.

The model can also account for the effects of the individual masking tones and the results on the nomask trials. The masking tone is assumed to add a constant, proportional to its duration, to the perceived duration of the target. As a result, a target followed by a mask after a long intertone interval will always appear longer than that same target on no-mask trials. Thus, both the long and the short 
targets will be categorized as short on a disproportionately large number of no-mask trials. This predicts the obtained results of high accuracy in identifying the short target and poor accuracy in identifying the long target on no-mask trials.

Finally, the perceived duration is influenced by the duration of the mask. A short target followed by a short mask will always have a shorter perceived duration than a short target followed by a long mask. Thus a greater proportion of short-target/long-mask combinations will exceed criterion and be erroneously categorized as long than will short-target/short-mask combinations. This will result in greater accuracy for identifying the short target when it is followed by the short mask. Similarly, a long target followed by a long mask will have a longer perceived duration than a long target followed by a short mask. Thus, a larger proportion of long-target/long-mask trials will exceed criterion and be categorized as long, ensuring greater accuracy of identification of the long target when followed by a long mask. This is precisely the form of the obtained target duration by mask duration interaction seen in Figures 4 and 5 . One final assumption is that the degree to which the mask lengthens the target's perceived duration is also a function of the duration of the target. Figure 5 shows that the effect of the duration of the mask on the target is greater for the short than for the long target. This assumption is compatible with Gol'dburt's (1961) finding of a greater effect of a mask on a short than on a long target.

In order to more precisely evaluate the fit of the model, we have developed a formalized version of the theory just given. The perceived duration (PD) of the target can be conceptualized as an exponential growth function of time:

$$
P D=\alpha\left(1-\mathrm{e}^{-\theta t}\right),
$$

where $\alpha$ is the asymptotic value of perceived duration and $\theta$ is the rate of growth of PD to this asymptote over time, $t$. More specifically, a represents the maximal perceived duration which is possible for a single tone, given infinite processing time. For targets as short as those used in the current studies, $\alpha$ will most probably represent an overestimate of the objective target durations (Efron, 1970 a, b, c). However, the present data indicate that these overestimates will not yield a constant perceived duration for the two targets, as suggested by Efron, but rather will be directly related to the actual temporal extents of the targets. This assumption is supported by recent work (Idson \& Massaro, Note 2) in which subjects were asked to rate the subjective durations of the target tones in Experiment 1 . In all conditions, the long target was rated as having a longer perceived duration than the short target, indicating that the perceived duration of the target was a direct function of its temporal extent.
When the mask is presented, it terminates further growth of the duration percept. Accordingly, processing time is indexed by the SOA values between the target and the mask. In addition to terminating the growth of the duration percept, the mask serves to lengthen the perceived duration of the target by a constant proportional to the actual duration of the mask. Incorporating this assumption into Equation 2 yields:

$$
\mathrm{PD}=\alpha\left(1-\mathrm{e}^{-\theta t}\right)+\mathrm{K}\left(\mathrm{t}_{\mathrm{m}}\right),
$$

where $t_{m}$ is the mask duration and $K$ is a constant of proportionality. If $P D_{S}$ and $P D_{L}$ represent the perceived durations of the short and the long targets, respectively, then the growth of the duration percept over time is given by:

$$
\begin{gathered}
P D_{S}=a_{S}\left(1-\mathrm{e}^{-\theta_{S} t}\right)+K_{S}\left(t_{m}\right) \\
P D_{L}=\alpha_{L}\left(1-e^{-\theta_{L} t}\right)+K_{L}\left(t_{m}\right),
\end{gathered}
$$

where $\theta_{\mathrm{S}}$ and $\theta_{\mathrm{L}}$ represent the rates of growth of the short and long targets to asymptotes $\alpha_{\mathrm{S}}$ and $\alpha_{\mathrm{L}}$, respectively, and $K_{S}$ and $K_{L}$ are the weights given the mask duration for the short and long targets, respectively. The discriminability between the two target durations would simply be equal to the difference between their perceived durations. Accordingly, subtracting Equation 4 from Equation 5 and rearranging terms yields a function for discriminability as indexed by $\mathrm{d}^{\prime}$,

$$
\mathrm{d}^{\prime}=\alpha_{\mathrm{L}}-\alpha_{\mathrm{S}}+\alpha_{\mathrm{S}} \mathrm{e}^{-\theta_{\mathrm{S}} \mathrm{t}}-\alpha_{\mathrm{L}} \mathrm{e}^{-\theta_{\mathrm{L}} \mathrm{t}}+\left(\mathrm{K}_{\mathrm{L}}-\mathrm{K}_{\mathrm{S}}\right) \mathrm{t}_{\mathrm{m}} \text {. }
$$

In order to determine asymptotic discriminability between the long and short tones, $t$ is made very large, driving the e terms to zero. This gives

$$
\mathrm{d}^{\prime}=\alpha_{\mathrm{L}}-\alpha_{\mathrm{S}}+\left(\mathrm{K}_{\mathrm{L}}-\mathrm{K}_{\mathrm{S}}\right)\left(\mathrm{t}_{\mathrm{m}}\right) .
$$

On no-mask trials, $t_{m}$ is zero, so that

$$
\mathrm{d}^{\prime}=\alpha_{\mathrm{L}}-\alpha_{\mathrm{S}}
$$

Equation 8 shows that asymptotic discriminability measured by $\mathrm{d}^{\prime}$ is equal to the difference between the asymptotic perceived durations (PDs) of the long and short tones. Equations 7 and 8 show that $d^{\prime}$ for an asymptotic masking condition can exceed the no-mask condition to the extent $K_{L}>K_{S}$. On the other hand, $d^{\prime}$ for the no-mask condition can exceed an asymptotic masking condition to the extent $K_{S}>K_{L}$.

It should be noted that Equation 6 is very similar in form to Massaro's (1975b) model of auditory recognition. 


$$
\mathrm{d}^{\prime}=\alpha\left(1-\mathrm{e}^{-\theta t}\right) .
$$

If $\theta_{\mathrm{S}}=\theta_{\mathrm{L}}=\theta$ and $\mathrm{K}_{\mathrm{L}}=\mathrm{K}_{\mathrm{S}}$, then Equation 6 reduces to

$$
\mathrm{d}^{\prime}=\left(\alpha_{\mathrm{L}}-\alpha_{\mathrm{S}}\right)\left(1-\mathrm{e}^{-\theta \mathrm{t}}\right),
$$

which can be made identical to Equation 9 by letting $\alpha_{\mathrm{L}}-\alpha_{\mathrm{S}}=\alpha$.

It should be noted that at very short ITIs, $t$ cannot be taken as an estimate of processing time due to an integration process at very short intervals (cf. Massaro, 1975b, Chapter 22). Accordingly, the observations taken at the 5-msec ITI will not be described by Equations 4, 5, and 6. These Equations predict that discriminability and perceived duration will both increase over time. At short ITIs, the tones should be perceived as relatively short and quite similar to one another. With increases in the ITI, perceived duration should increase to different asymptotes for the target tones, increasing the discriminability of the targets.

In order to apply this model to the obtained data, the assumption is made that noise in the sensory system creates a distribution of perceived durations for each experimental condition. The noise distribution is assumed to be normal with a variance equal to 1 . The data were first transformed into relative standard deviation units, which provide an index of perceived duration. In the context of the current model, the probability of responding long is a measure of the perceived duration of the target. Thus, the probability of responding long given a long target, $P(L \mid L)$, and the probability of responding long given a short target, $P(L \mid S)$, were computed at each experimental condition. A value of $P(L \mid L)$ or $P(L \mid S)$ can be used to determine the location of the mean of the subjective durations at that condition with respect to the fixed criterion value. The $\mathrm{z}$ distance between the mean of the relevant distribution and the criterion value can, therefore, be computed for both the long and the short targets. If $P(L \mid L)$ or $P(L \mid S)$ is greater than .5, this indicates that the mean of the distribution is to the right of the criterion. In this case, the $\mathrm{z}$ distance will be positive and will correspond to the proportion of the distribution between the mean and criterion value. If $P(L \mid L)$ or $P(L \mid S)$ is less than .5, the mean is to the left of the criterion and the $z$ distance will be negative and will correspond to the proportion of the distribution between the mean and the criterion value. In all cases, the $z$ distance will be simply the transform of the proportion of the long or short distribution lying between the mean and the criterion (cf. Massaro, 1975b, for a more complete discussion and a table of $z$ distances). In order to ensure that all scores were positive, so that Equations 4 and 5 could be applied, the value of +3 was added to all of the $z$ values.

The model was then fit to these values of $z[P(L \mid L)]$ and $z[P(L \mid S)]$ using the reiterative computer program STEPIT (Chandler, 1969). The 5-msec intertone interval was excluded from the fit, as a process of integration which occurs at this interval precludes direct application of the model (Massaro, 1975b; Massaro et al., 1976). SOA values were used in applying Equations 4 and 5. For the long target, the SOAs were $115,135,195,255,295$, and $345 \mathrm{msec}$, while for the short target, the SOAs were $75,95,155$, 215,255 , and $305 \mathrm{msec}$.

Figure 10 presents the predicted and observed values of $z[P(L \mid L)]$ and $z[P(L \mid S)]$. The predicted values were computed by estimating the parameter values $\alpha_{\mathrm{L}}, \alpha_{\mathrm{S}}, \theta_{\mathrm{L}}, \theta_{\mathrm{S}}, \mathrm{K}_{\mathrm{L}}$, and $\mathrm{K}_{\mathrm{S}}$ by minimizing the squared deviations between the predicted and observed values. As can be seen from the figure, the model provides a relatively good fit to the data, considering that 38 data points $(2$ targets $\times 3$
Figure 10. Observed and predicted values of $z[P(L \mid L)]$ and $z[P(L \mid S)]$ as a function of both the duration of the masking tone and the intertone interval. The left-hand panel displays the data for the short masking tone, the middle panel for the mid masking tone, and the right-hand panel for the long masking tone. The no-mask values do not differ as a function of masking tone duration, since the latter is a dummy variable under the nomask condition (Experiment 1).

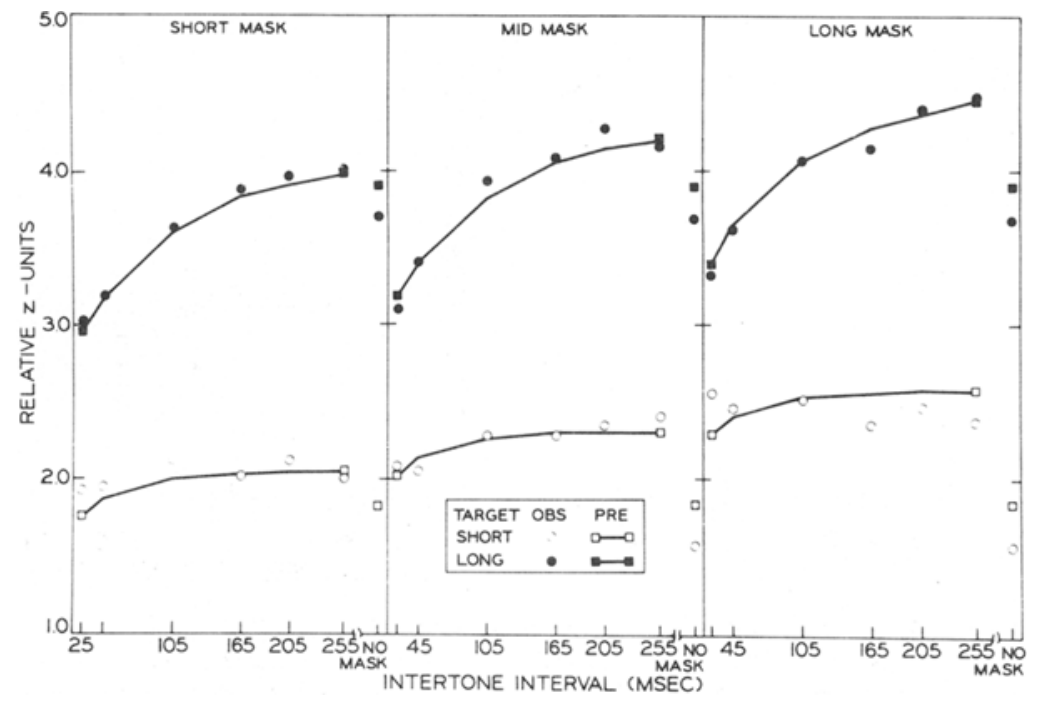


masks $\times 6$ intertone intervals +2 no-mask targets) were fit with just six parameters. The average squared deviation between the predicted and the observed values was .011. The parameter estimates for $\alpha_{S}$ and $\alpha_{L}$ were 1.85 and 3.90, respectively. These parameter estimates were in accord with the model, the long target having a greater potential perceived duration than the short target. The parameter estimates for $\theta_{\mathrm{L}}$ and $\theta_{\mathrm{S}}$ were 10.95 and 25.00 , respectively. Processing of the short target, therefore, occurred at a faster rate than the long target. The parameter estimates for $\mathrm{K}_{\mathrm{L}}$ and $\mathrm{K}_{\mathrm{S}}$ were 5.69 and 6.73 , respectively. This indicates that the duration of the mask had a slightly greater effect on the perceived duration of the short than of the long target.

The model provides a good description of the changes in perceived duration in Experiment 1 as a function of the target duration, mask duration, and the ITI. One critical property of the model is the distinction between the growth of the duration percept that is terminated by the onset of the masking tone and the additional contribution of the masking tone when it is incorporated into the perceptual judgment. The two contributions are given by the first and second terms contributing to PD in Equation 3. A direct test of this property is provided by the long ITIs employed in Experiment 5 . We assumed that the growth of the duration percept should reach $\alpha$ at roughly the same ITI as in Experiment 1, but that the masking tone can be incorporated into the judgments at even longer ITIs. Furthermore, subjects should be more likely to make a judgment before the mask is presented as the ITI becomes sufficiently long. We assumed that the masking tone was always incorporated into the judgment at ITIs of $255 \mathrm{msec}$ or less and on only a proportion of the trials at the longer ITIs. Performance at these longer ITIs was, therefore, a mixture of "masking" and "no-mask" trials. In the quantitative description of the results, this proportion was estimated as a free parameter for each of the ITIs of 505, 755, and 1,005 msec. Otherwise, the quantitative fitting procedure was equivalent to that used on the results in Experiment 1. .

Figure 11 presents the predicted and observed values of $z[P(L \mid L)]$ and $z[P(L \mid S)]$. The close

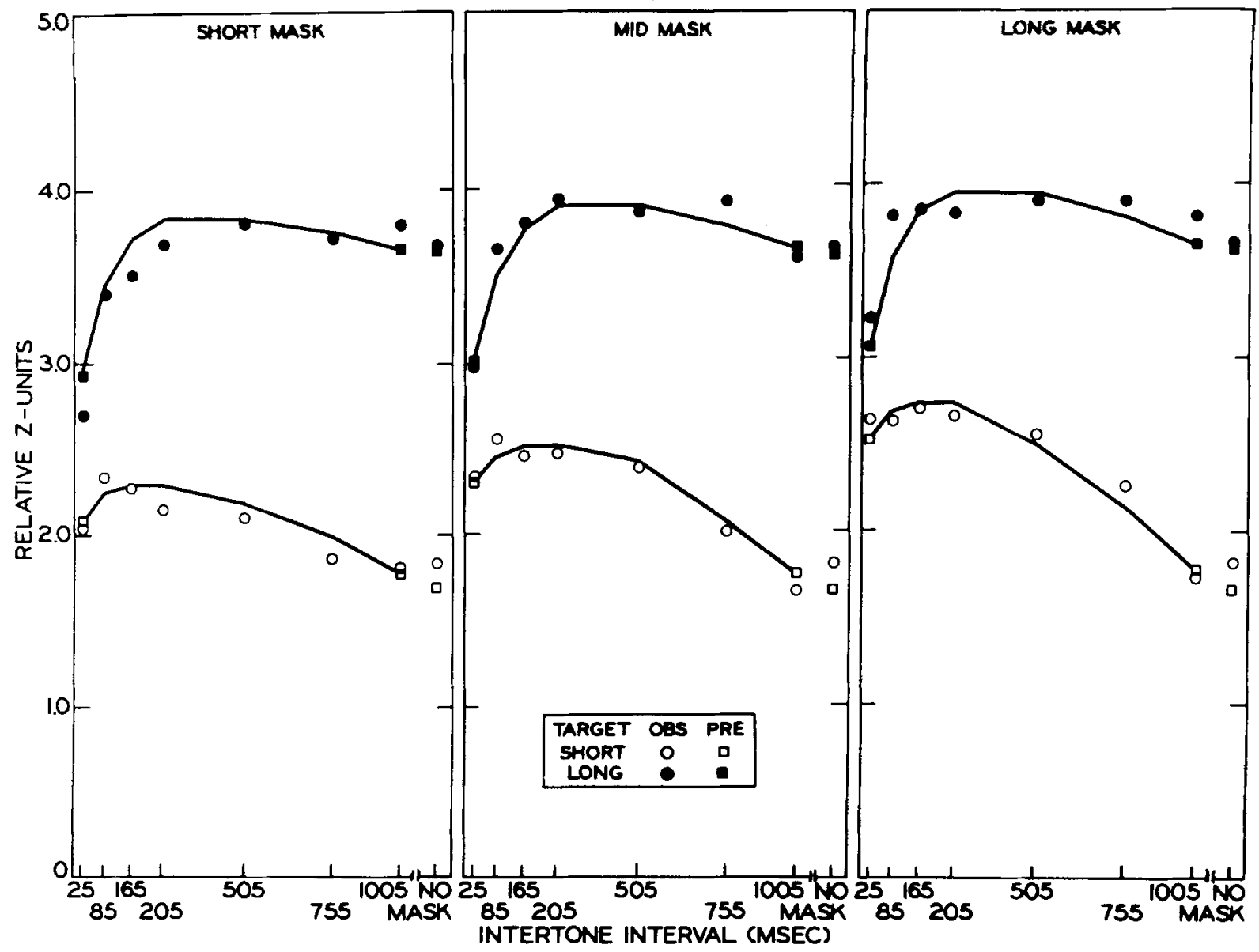

Figure 11. Observed and predicted values of $z[P(L \mid L)]$ and $z[P(L \mid S)]$ as a function of both the duration of the masking tone and the intertone interval. The left-hand panel displays the data for the short masking tone, the middle panel for the mid masking tone, and the right-hand panel for the long masking tone. The no-mask values do not differ as a function of masking tone duration, since the latter is a dummy variable under the no-mask condition (Experiment 5). 
correspondence between predicted and observed values supports our interpretation of the long ITIs in Experiment 5. The squared deviation between predicted and observed values averaged .020. The parameter values for $\alpha_{\mathrm{L}}, \alpha_{\mathrm{S}}, \theta_{\mathrm{L}}$, and $\theta_{\mathrm{S}}$ were 3.64 , $1.69,12.74$, and 25.00 , respectively. These values were very similar to those found in Experiment 1 . The parameter estimates for $K_{L}$ and $K_{S}$ were 4.57 and 11.71 , respectively. This indicates that the duration of the mask had a much smaller effect on the perceived duration of the long than of the short target. The other three parameter estimates indicated that the masking tone was incorporated into the duration judgment on $.80, .46$, and .10 of the trials for the ITIs of 505,755 , and 1,005 , respectively.

It should be noted that $d^{\prime}$, as measured by the difference between $z[P(L \mid L)]$ and $z[P(L \mid S)]$ continued to increase with increases in the ITI beyond $255 \mathrm{msec}$ even though the growth of the duration percept with processing time had already reached the asymptote $\alpha$. This result can be seen by the predictions of Equations 7 and 8; the larger value for $K_{S}$ than for $K_{L}$ decreases performance on masking relative to no-mask trials. Assuming that the masking tone is incorporated into the duration judgment on a smaller proportion of the trials as the ITI is increased results in the corresponding increase in $d^{\prime}$ with increases in ITI. This result supports the distinction between the dynamic grcwth of perceived duration as indexed by the first term in Equation 3 and the additional contribution of the masking tone as indexed by the second term of Equation 3.

The present results and theoretical analysis also appear to be relevant to visual processing. Similar results have been obtained by Cantor and Thomas (1976) in a visual masking study. A target stimulus was presented for 30 or $50 \mathrm{msec}$, followed after an ISI of $0,30,70$, or $110 \mathrm{msec}$ by a 500 -msec presentation of a masking field. The target stimuli were either two circles of differing sizes or two different abstract forms. In separate sessions, the subjects were asked to judge the duration, size (circles), or form (abstract forms) of the target stimuli. Accuracy of discrimination of size and form increased monotonically with increases in both stimulus duration and the ISI, and the perceived duration of the form stimuli increased directly with increases in stimulus duration and ISI. These results are largely predictable from the model presented here. The monotonic increase in accuracy with increases in stimulus duration and ISI can be described directly by Equation 1. More important, the increase in perceived duration for the form stimuli with increases in processing time replicates the current results and can be interpreted in a similar manner. It should be noted, however, that Thomas and Cantor (1976; Cantor \& Thomas, 1976) have developed a somewhat different model to account for these results. In many respects, their model is comparable to that presented here, in that they also assume that perceived duration increases with increases in processing time. The key difference is that in the Thomas and Cantor model the duration percept is assumed to be derived from the time spent processing the nontemporal information in the stimulus. As processing time for nontemporal information will increase with increases in SOA and perceived duration is assumed to be directly related to processing time, this model can also predict the obtained results. Though it should be possible to distinguish empirically between these two models, their overall similarity reinforces the idea of analogous dynamic mechanisms in visual and auditory information processing.

The backward masking results and theoretical analysis suggests an explanation for certain previous findings in this area. If the perception of tonal duration grows over time, then the decrement in discriminability found in a two-interval forcedchoice task with short interstimulus intervals (Carbotte, 1973; Small \& Campbell, 1962) is predictable. With less than a separation of approximately $250 \mathrm{msec}$ between the tones in the two intervals of the forced-choice task, the second tone can be conceptualized as masking the perceived duration of the first. Massaro and Idson (Note 1) have, in fact, demonstrated backward masking effects in a two-interval forced-choice task, lending credence to this interpretation.

In summary, the extension of Massaro's (1975b) model of auditory recognition has described perceived duration in the same conceptual framework as other attributes of sound. It was necessary, however, to distinguish between two measures of perceptual behavior, accuracy and perceptual experience. Both of these measures are necessary to capture the important aspects of how the duration of sound is processed. Our goal is to extend previous work using accuracy measures in backward recognition masking to include perceptual experience measures of processing other dimensions of sound.

\section{REFERENCE NOTES}

1. Massaro, D. W., \& Idson, W. L. Backward recognition masking of relative pitch judgments. Unpublished manuscript, 1975.

2. Idson, W. L., \& Massaro, D. W. Perceptual processing and experience of auditory duration. Unpublished manuscript, 1976.

\section{REFERENCES}

AreL, S. M. Duration discrimination of noise and tone bursts. Journal of the Acoustical Society of America, 1972, 15, 12191223. (a)

AвEL, S. M. Discrimination of temporal gaps. Joumal of the Acoustical Society of America, 1972, 52, 519-524. (b)

Allan, L. G., \& Kristofferson, A. B. Successiveness discrimination: Two models. Perception \& Psychophysics, 1974, 15, 37-46. (a) 
Allan, L. G.. \& Kristofperson, A. B. Psychophysical theories of duration discrimination. Perception \& Psychophysics. 1974. 16. 26-34. (b)

Allan, L. G.. \& Kristofferson, A. B. Judgments about the duration of brief stimuli. Perception \& Psychophysics, 1974. 15. 434-440. (c)

Allan, L. G.. Kristofferson. A. B.. \& Rice, M. E. Some aspects of perceptual coding of duration in visual discrimination. Perception \& Psychophy'sics. 1974. 15. 83-88.

Allan, L. G.. Kristofferson, A. B.. \& Wiens, E. W. Duration discrimination of brief light flashes. Perception \& Psychophysics, 1971, 9. 327-334.

Cantor. N. E., \& Thomas, E. A. C. Visual masking effects on duration. size. and form discrimination. Perception \& Psychophysics. 1976, 19.321-327.

Carbotte. R. M. Retention of time information in forced-choice duration discrimination. Perception \& Psychophysics. 1973. 14. 440-444.

Chandler, J. P. Subroutine STEPIT-Finds local minima of a smooth function of several parameters. Behavioral Science, 1969. 14. 81-82.

Creelman. C. D. Human discrimination of auditory duration. Journal of the Acoustical Society of A merica, 1962, 34, 582-593.

DE UTSCH. D. Tones and numbers: Specificity of interference in immediate memory. Science, 1970. 168. 1604-1605.

EFRON. R. Effects of stimulus duration on perceptual onset and offset latencies. Perception \& Psychophysics, 1970, 8. 231-234. (a)

Efron. R. The measurement of perceptual duration. Studium Generale. 1970. 23. 550-561. (b)

Efron. R. The minimum duration of a perception. Neuropsychologia. 1970, 8, 57-63. (c)

GOL'DBURT. S. N. Investigation of the stability of auditory processes in micro-intervals of time (new findings in back masking). Biophysics. 1961, 6, 809-817.

GreEn. D. M., \& Swets. J. A. Signal detection theory and psychophyisics. New York: Wiley, 1966.

Hawkins, H. L., Thomas, G.. Presson, J.. Cozic, A., \& BROOKMIRE, D. Tonal specificity and masking in auditory recog. nition. Joumal of Experimental Psychology, 1974, 103, 530-538.
Hawkins. H. L., \& Presson, J. C. Masking and preperceptual selectivity in auditory recognition. In S. Dornic (Ed.), Attention and performance VI. New York: Academic Press, in press.

Massaro, D. W. Preperceptual auditory images. Joumal of Experimental Psychology. 1970. 85. 411.417. (a)

MASSARO. D. W. Retroactive interference in short-term recognition memory for pitch. Journal of Experimental Psychology, 1970, 83, 32-39. (b)

Massaro, D. W. Preperceptual images, processing time, and perceptual units. Psychological Review, 1972, 79. 124-145. (a)

Massaro. D. W. Stimulus information versus processing time in auditory pattern recognition. Perception \& Psychophysics, 1972 , 12. 50-56. (b)

Massaro. D. W. Perceptual units in speech recognition. Joumal of Experimental Psychology. 1974. 102. 199-208.

MASSARO. D. W. Backward recognition masking. Joumal of the Acoustical Society of America, 1975. 58. 1059-1065. (a)

MAsSARO. D. W. Experimental psychology and information processing. Chicago: Rand-McNally. 1975. (b)

Massaro, D. W., Cohen, M. M., \& IDSON, W. L. Recognition masking of auditory lateralization and pitch judgments. Journal of the Acoustical Society of America, 1976, 59, 434-441.

Massaro, D. W.. \& KaHN. B. Effects of central processing on auditory recognition. Joumal of Experimental Psychology, 1973. 97. $51-58$.

Moore. J. J., \& MAssaro, D. W. Attention and processing capacity in auditory recognition. Joumal of Experimental Psychology. 1973. 99. 49-54.

Small. A. M.. \& Campbell, R. A. Temporal differences in sensitivity for auditory stimuli. American Journal of Psychology, 1962 , 75. 401-410.

Thomas, E. A. C., \& CANTor. N. E. Simultaneous time and size perception. Perception \& Psychophysics, 1976, 19. 353-360.

WOLF. C. G. A recognition masking study of consonant processing. Perception \& Psychophysics, 1976. 19. 35-46.

(Received for publication March 22, 1976: revision received July 28. 1976.) 